ARTICLE

https://doi.org/10.1038/s41467-019-13639-8

\title{
Stress experience and hormone feedback tune distinct components of hypothalamic $\mathrm{CRH}$ neuron activity
}

\author{
Joon S. Kim (1) ${ }^{1}$, Su Young $\operatorname{Han}^{1} \&$ Karl J. Iremonger (iD ${ }^{1 \star}$
}

Stress leaves a lasting impression on an organism and reshapes future responses. However, the influence of past experience and stress hormones on the activity of neural stress circuits remains unclear. Hypothalamic corticotropin-releasing hormone $(\mathrm{CRH})$ neurons orchestrate behavioral and endocrine responses to stress and are themselves highly sensitive to corticosteroid (CORT) stress hormones. Here, using in vivo optical recordings, we find that $\mathrm{CRH}$ neurons are rapidly activated in response to stress. $\mathrm{CRH}$ neuron activity robustly habituates to repeated presentations of the same, but not novel stressors. CORT feedback has little effect on $\mathrm{CRH}$ neuron responses to acute stress, or on habituation to repeated stressors. Rather, CORT preferentially inhibits tonic CRH neuron activity in the absence of stress stimuli. These findings reveal how stress experience and stress hormones modulate distinct components of $\mathrm{CRH}$ neuronal activity to mediate stress-induced adaptations.

\footnotetext{
${ }^{1}$ School of Biomedical Sciences, Centre for Neuroendocrinology and Department of Physiology, University of Otago, Dunedin 9054, New Zealand. *email: karl.iremonger@otago.ac.nz
} 
S tress is a collective term encompassing the repertoire of neural, endocrine, and physiological responses an organism mounts in the face of threat ${ }^{1}$. Stressful experiences evoke a cascade of hormonal and neural changes to promote adaptation and reshape future responses ${ }^{2,3}$. It has long been appreciated that neural circuits in the hypothalamus are essential for coordinating organism's responses to real or perceived threats ${ }^{4-6}$. One hypothalamic neural population, which are essential for controlling stress responses, are the corticotropin-releasing hormone (CRH) neurons located in the paraventricular nucleus (PVN). In addition to their well-known role in controlling corticosteroid (CORT) secretion, this neural population has also been shown to be important in mediating other stress-related functions, including shifts in behavior ${ }^{7}$, pheromone release ${ }^{8}$, and encoding of valence ${ }^{9}$. Furthermore, prior stress experience can modify subsequent behavioral and endocrine output ${ }^{7,10-12}$, suggesting that $\mathrm{CRH}$ neuron responses may be highly adaptable.

Two broad forms of plasticity are thought to regulate $\mathrm{CRH}$ neuron responses to stress: $2,13,14$ plasticity induced by activation of stress hormone receptors and plasticity induced by stressevoked neural activity. Stress-induced CORT elevations have long been theorized to feedback to the brain and acutely "shut-off" $\mathrm{CRH}$ neuron activity and limit future stress responses ${ }^{2,14,15}$. Although past in vitro experiments have clearly shown that elevations in CORT can modify $\mathrm{CRH}$ neuron cellular function and excitability ${ }^{16-18}$, evidence that these can drive changes in excitability in vivo are lacking. Neurally driven plasticity, which is not stress hormone dependent, has also been demonstrated in vitro ${ }^{19-21}$. Yet, how this type of plasticity shapes in vivo $\mathrm{CRH}$ neuron responses is also unclear.

While neurally driven plasticity following stress should be induced quickly, plasticity driven by stress hormone signaling inherently possess a temporal delay in vivo ${ }^{22}$. Thus, neural and hormonal mechanisms would be anticipated to mediate distinct forms of stress-induced adaptation in neural circuits. We therefore set out to understand how stress experience and stress hormone signaling regulate $\mathrm{CRH}$ neural activity in vivo. We report that hypothalamic CRH neurons are tonically active in vivo and rapidly respond to threat. Repeated exposure to homotypic stress suppressed threat-evoked CRH neuron activity over a time course of minutes to days. Importantly, this adaptive response did not require stress hormone signaling. While CORT feedback had no effect on the magnitude of threat-evoked activity, it did induce a slow suppression of tonic CRH neural activity. Together, these data reveal that neural and endocrine mechanisms regulate different components of hypothalamic CRH neuron activity dynamics.

\section{Results}

Optical recordings of CRH neuron activity in vivo. To gain insight into how neural activity in the hypothalamic $\mathrm{CRH}$ neuron population is regulated by stress, we performed GCaMP6s fiber photometry (Fig. 1b) in freely behaving adult male Crh-IRES-cre mice ${ }^{23}$. Dual in vitro loose patch recordings and confocal GCaMP6s imaging showed a high correlation between spiking activity and changes in GCaMP6s fluorescence (Supplementary Fig. 1B-E), confirming that GCaMP6s reliably reports the spiking activity of CRH neurons.

We then characterized the dynamics of $\mathrm{CRH}$ neuron population activity in vivo using fiber photometry in resting, stressed, and post-stress conditions (see Supplementary Movie 1 and Fig. 1e, f). In the absence of an external threat stimulus, the $\mathrm{CRH}$ neuron population exhibited tonic activity consisting of low irregular GCaMP6s transients (Fig. 1e, f). Under these conditions, circulating CORT levels remained low (samples taken before $(33.6 \pm 2.4 \mathrm{ng} / \mathrm{mL}, n=8$; mean \pm SEM $)$ and after $(36.2 \pm 1.8 \mathrm{ng} / \mathrm{mL}) 90 \mathrm{~min}$ in test apparatus; Fig. 1c). The baseline activity dynamics were not caused by movement or light artifacts as they were not observed in the $405 \mathrm{~nm}$ reference channel (Supplementary Fig. 2B) or in control mice expressing GFP (Supplementary Fig. 2C).

Across most experiments, we used a loud white noise $(85 \mathrm{~dB}, 5$ $\mathrm{min}$ ) to induce stress-evoked $\mathrm{CRH}$ neuron activation. This type of stressor has been extensively used ${ }^{24-26}$ and has the advantage of being highly stereotyped, repeatable, and does not require the experimenter to physically manipulate the animal. CRH neurons were strikingly responsive to this stress stimulus, behaving like a neuronal alarm system as recently reported ${ }^{9,27}$. A strong response was observed almost immediately following the white noise onset, which was followed by a sustained elevation of neural activity during the noise exposure (stress-induced activity). The white noise-induced peak $\Delta F / F$ was $0.89 \pm 0.08(n=64$; Fig. $1 \mathrm{~g})$ and average time to peak from white noise onset was $4.8 \pm 0.32 \mathrm{~s}(n=$ 64; Fig. 1h). Mean increase in GCaMP fluorescence during the 5 min white noise exposure was $0.39 \pm 0.03 \Delta F / F$ ( $n=64$; Fig. 1i). White noise also robustly increased circulating CORT levels (samples taken $60 \mathrm{~min}$ before $(33.5 \pm 2.2 \mathrm{ng} / \mathrm{mL}$ ) and $30 \mathrm{~min}$ post $(147.5 \pm 10.2 \mathrm{ng} / \mathrm{mL})$ white noise stress, $n=8, p<0.001$ paired $t$ test; Fig. 1d).

Interestingly, we observed variability in the CRH neuron activity off-set kinetics after the $5 \mathrm{~min}$ white noise (post-stress activity). Some mice exhibited total shut down of activity (return to baseline) almost immediately after the cessation of the white noise (Fig. 1, $\mathrm{F}_{1-3}$ ), while others displayed elevated irregular or sustained activity during the post-stress period (Fig. 1, $\mathrm{E}_{1-3}$ ). When all responses were averaged together, CRH neuron activity returned to baseline levels $(p>0.05$, repeated-measures (RM) one-way analysis of variance (ANOVA)) at $3.8 \mathrm{~min}$ post stress (Fig. 1j). Taken together, these data show that $\mathrm{CRH}$ neurons are a vigilant neuronal population that exhibit dynamic responses to novel threat.

CRH neurons adapt to familiar stress independent of CORT. We next sought to address how adaptions to stress-evoked $\mathrm{CRH}$ neuron responses are tuned by stress hormone feedback. Given the well-described adaptive properties of CORT for learning and stress habituation, we designed a sequential stress protocol to observe the effects of endogenous CORT-negative feedback on $\mathrm{CRH}$ activity and stress adaptation. In these experiments, we presented two identical white noise stressors (WN1 and WN2) separated by $30 \mathrm{~min}$ ( $n=13$ per group; Fig. 2$)$ or $120 \mathrm{~min}(n=11$ per group; Fig. 3) to mice treated with either vehicle or the CORT synthesis inhibitor, metyrapone.

Following an initial large response to the first white noise (WN1), vehicle-treated mice gradually reduced their $\mathrm{CRH}$ neural activity down to basal levels $(n=13$; Fig. $2 \mathrm{a}, \mathrm{b})$. When a second white noise (WN2) was applied following a 30-min interval, both the peak $\Delta F / F$ response (Veh-WN1 $1.0 \pm 0.14 \Delta F / F$ vs. Veh-WN2 $0.87 \pm 0.12 \Delta F / F, p=0.03 \mathrm{RM}$ two-way ANOVA; Fig. $2 \mathrm{c})$ and the mean $\triangle F / F$ response (mean $C R H$ activity during $5 \mathrm{~min} W \mathrm{~W}$ : VehWN1 $0.42 \pm 0.07 \Delta F / F$ vs. Veh-WN2 $0.27 \pm 0.05 \Delta F / F, p<0.001$ RM two-way ANOVA; Fig. 2d) were significantly reduced in the vehicle-treated group. We hypothesized that elevated CORT levels consequent from WN1 (Fig. 1d) may mediate the reduced $\mathrm{CRH}$ neuron excitability during WN2.

To address the role of CORT feedback in this adaptive change, metyrapone was used to inhibit CORT synthesis (samples taken $60 \mathrm{~min}$ before $(35.3 \pm 2.7 \mathrm{ng} / \mathrm{mL})$ and $30 \mathrm{~min}$ post $(39.7 \pm$ $5.0 \mathrm{ng} / \mathrm{mL}$ ) WN, $n=8, p=0.5$ paired $t$ test; Fig. $2 \mathrm{e})$. Responses to $\mathrm{WN} 1$ and the initial post-stress kinetics were virtually identical between the vehicle $(0.42 \pm 0.07$ and $0.15 \pm 0.04 \Delta F / F, 5$ min mean 

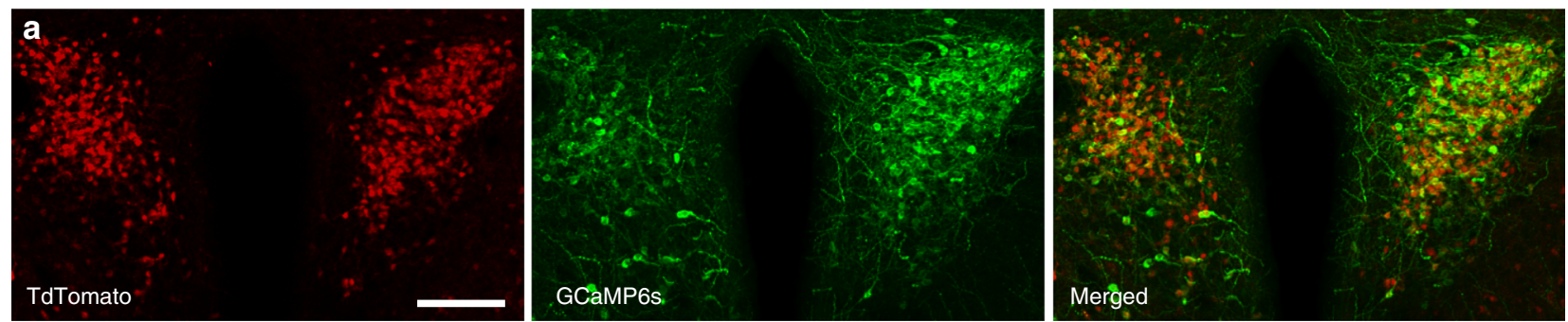

b
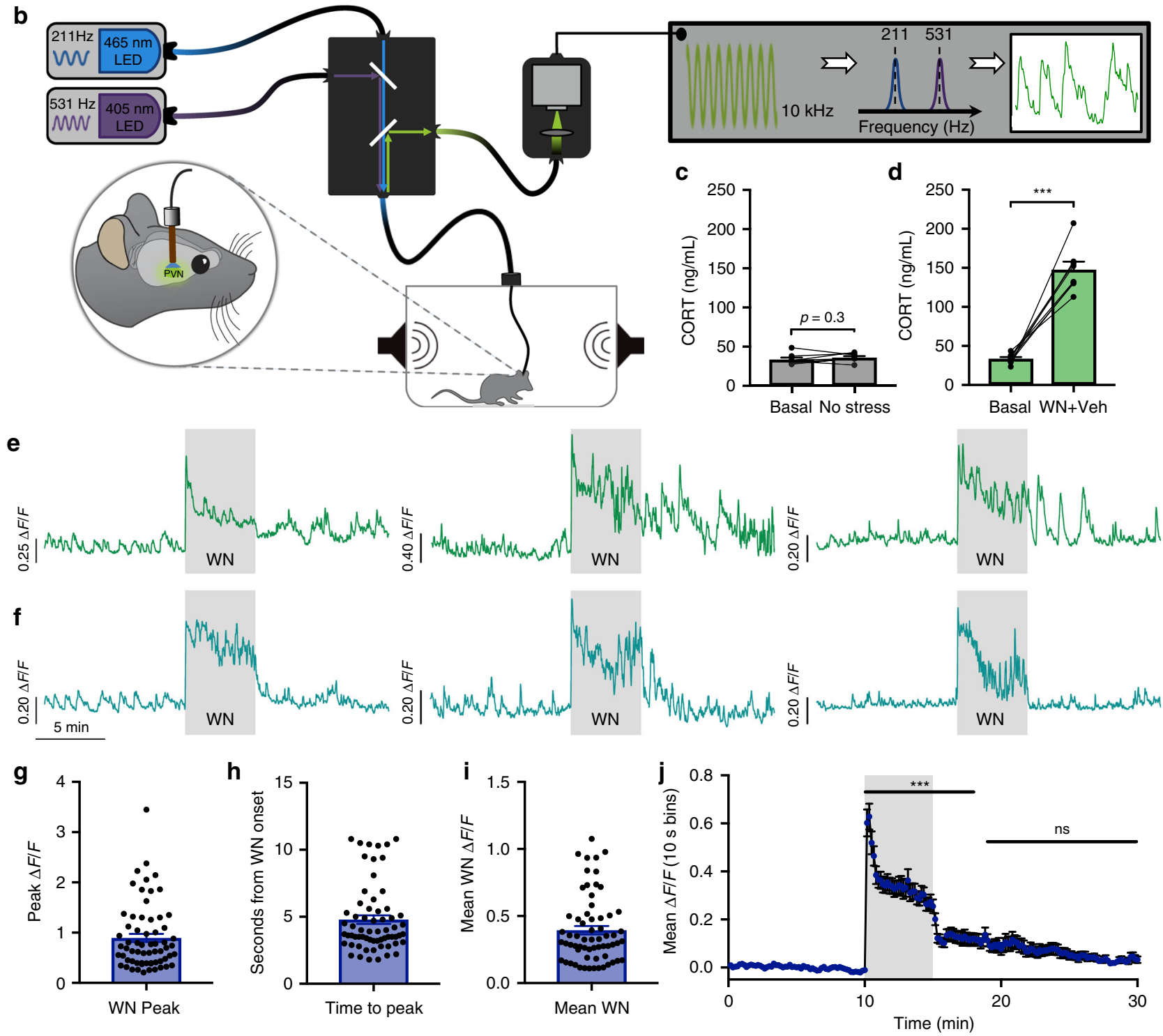

Fig. 1 Optical recordings of CRH neuron activity in freely behaving mice. a Image showing PVN expression of CRH-tdTomato reporter (left), AAV-driven GCaMP6s (center), and merged image (right). Scale bar: $100 \mu \mathrm{m}$. b Schematic illustration of the fiber photometry setup. c Blood CORT concentrations obtained from tail tip samples, while mice remained within experimental testing box in the absence of white noise (WN) stress; $n=8, p>0.05$, paired $t$ test. d Blood CORT concentrations following WN stress; $n=8,{ }^{\star \star *} p<0.001$, paired $t$ test. e Photometry recordings of CRH neurons from three individual mice displaying continued activity after termination of WN. $\mathbf{f}$ Photometry recordings of $\mathrm{CRH}$ neurons from three individual mice displaying rapid cessation of activity after termination of WN. $\mathbf{g}$ Peak $\Delta F / F$ at WN stress onset, $n=64$. $\mathbf{h}$ Time to peak from the onset of WN onset, $n=64$. i Mean $\Delta F / F$ of CRH neuron activity during 5 min WN stress from all individual mice tested, $n=64$. j Mean CRH neuron $\Delta F / F$ in 10 sec bins from all individual mice; $n=64$, repeated-measures (RM) one-way ANOVA ${ }^{\star \star \star} p<0.001$ vs. baseline, Dunn's post hoc test. All data are presented as mean \pm SEM.

$\mathrm{CRH}$ activity during and after white noise (WN1), respectively; Fig. 2d) and metyrapone-treated groups $(0.43 \pm 0.07$ and $0.19 \pm$ $0.04 \Delta F / F$, during and after WN1 respectively; Fig. 2d).

Mean CRH neural activity during WN2 was also not different between vehicle and metyrapone treatment groups (mean $\mathrm{CRH}$ activity during 5 min WN2: Veh-WN2 $0.27 \pm 0.05 \Delta F / F$ vs. METWN2 $0.33 \pm 0.05 \Delta F / F, p=0.37$ RM two-way ANOVA; Fig. 2d). Furthermore, the mean suppression of $\mathrm{WN} 2$ relative to $\mathrm{WN} 1$ was not significantly different between treatment groups (Veh-WN2 $65.4 \pm 6.2 \%$ of $\mathrm{WN1}$ response vs. MET-WN2 $81.5 \pm 8.2 \%$ of 
a

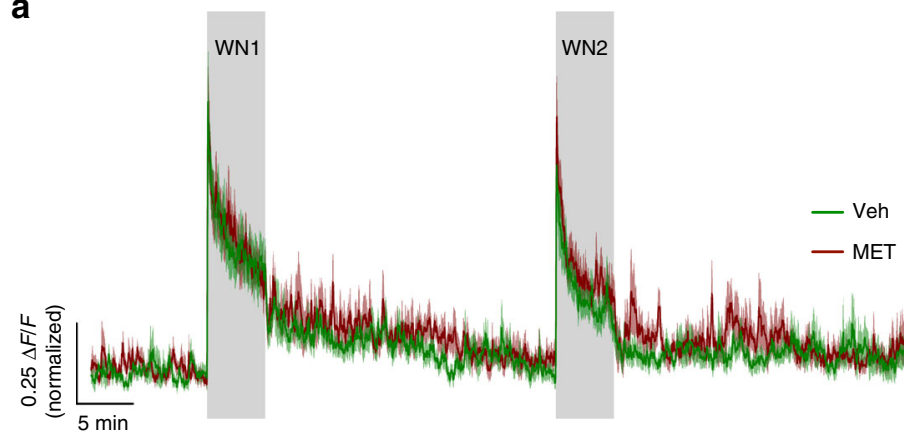

b

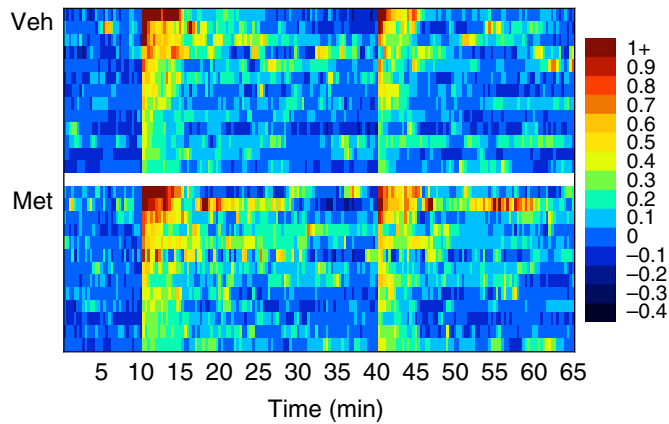

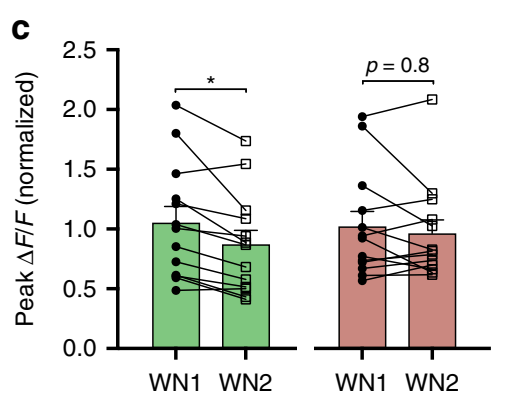
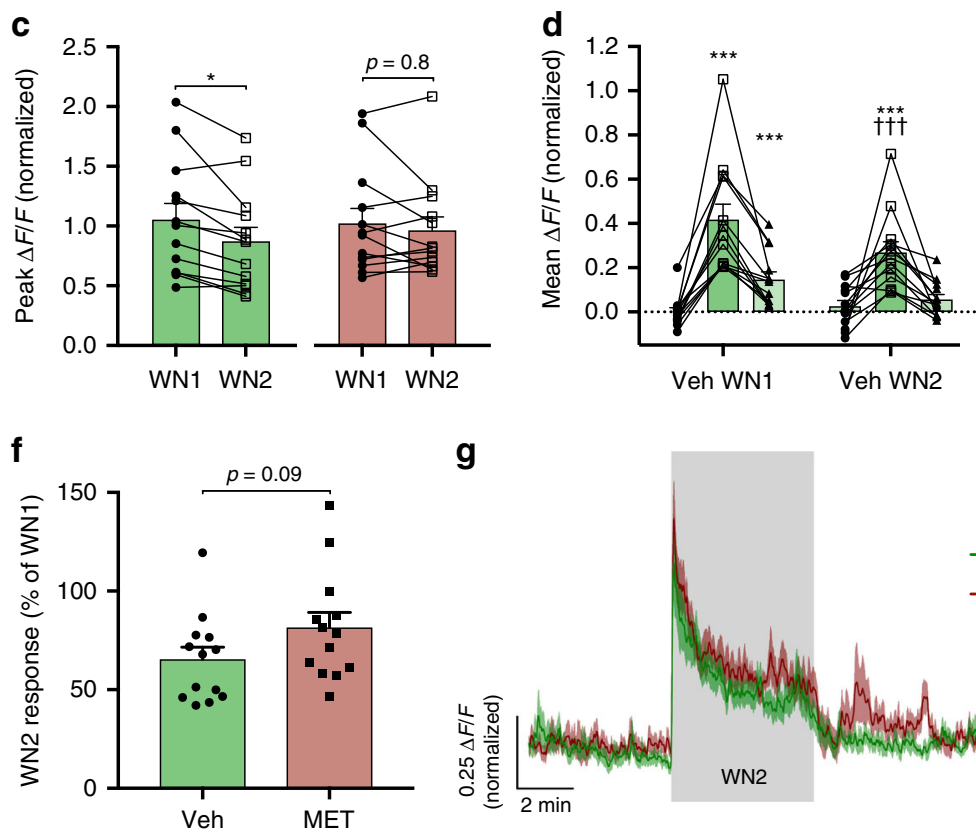

g

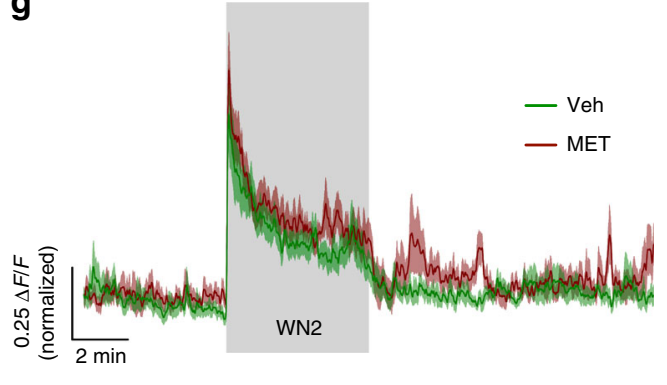

h

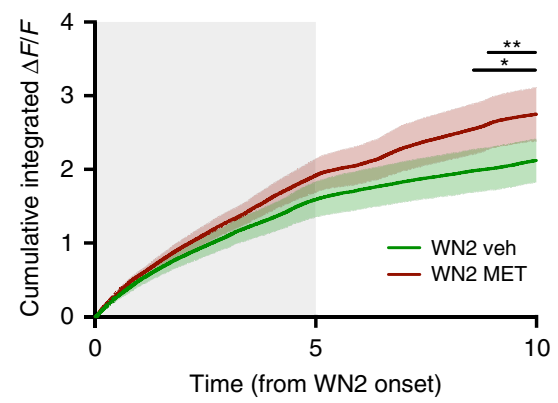

Fig. 2 CRH neuron shut-off and adaptation occur regardless of fast CORT feedback. a Mean photometry signals of CRH neuron activity induced by two sequential WN stressors 30 min apart from vehicle and metyrapone-treated mice. b Heatmap of mean CRH neuron activity from all individual mice in $20 \mathrm{~s}$ bins. c Peak $\Delta F / F$ at $W N$ onset; RM two-way ANOVA, ${ }^{*} p<0.05$ vs. WN1, Holm-Sidak; ANOVA interaction $p=0.14$, ANOVA main effect of group $p=0.86$. d Average $\Delta F / F$ across 5 min of $C R H$ neuron activity before, during, and after each $W N ; n=13$ per group, RM two-way ANOVA, ${ }^{*} p<0.05$ vs. baseline, ${ }^{\dagger} p<0.05$ vs. respective WN1 timepoint, Holm-Sidak; ANOVA interaction $p=0.90$, ANOVA main effect of group $p=0.38$. e CORT levels before and after WN stress following metyrapone treatment; $n=8$, paired $t$ test. f Percentages of CRH neuron activity during WN2 relative to WN1; Veh vs. MET, Mann-Whitney test. $\mathbf{g}$ Averaged photometry recordings of $\mathrm{CRH}$ neuron activity from all vehicle and metyrapone-treated mice showing the response to WN2. h Cumulative integrated $\Delta F / F$ from the time of WN2 onset; RM two-way ANOVA, ${ }^{\star} p<0.05$ Veh vs. MET, Holm-Sidak; ANOVA interaction $p<0.001$, ANOVA main effect of group $p=0.09$. Lines indicate points at which statistical significance was reached and its duration. Gray shaded area

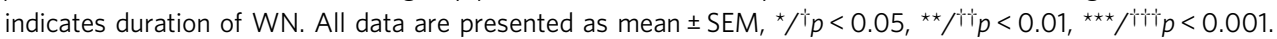

WN1, $p=0.09$ Mann-Whitney test; Fig. 2f). It was evident, however, that metyrapone-treated mice appeared to show a marginally elevated level of activity during and after WN2 (Fig. $2 \mathrm{~g}$ ). We therefore analyzed the cumulative $\Delta F / F$ during both the stress response and post-stress periods to detect changes in neural activity that manifest more slowly over time. Indeed, when the cumulative integrated $\Delta F / F$ was compared between groups, metyrapone-treated mice had a higher level of cumulative activity, which reached significance $3.5 \mathrm{~min}$ following the termination of WN2 (Veh vs. MET cumulative $\Delta F / F, p<0.05$ at $8.5 \mathrm{~min}$ from WN2 onset, RM two-way ANOVA; Fig. 2h). While no significant differences in mean or cumulative $\Delta F / F$ responses were observed during stress, loss of negative feedback led to slightly elevated activity that became evident in the poststress period.

These small differences in activity became more apparent when we applied a $120 \mathrm{~min}$ inter-stress interval $(n=11$ per group;
Fig. 3a, b). Vehicle and metyrapone-treated mice again exhibited similar responses to WN1 (Veh $0.36 \pm 0.06$ and $0.16 \pm 0.05 \Delta F / F$, 5 min mean $\mathrm{CRH}$ activity during and after $\mathrm{WN1}$, respectively; Fig. 3c; MET $0.38 \pm 0.05$ and $0.14 \pm 0.02 \Delta F / F$, during and after $\mathrm{WN1}$, respectively; Fig. 3c). However, significant differences in tonic activity became discernible $30 \mathrm{~min}$ post WN1 stress. Vehicle-treated mice exhibited a near-complete shut-off in $\mathrm{CRH}$ neuron activity whereas metyrapone-treated mice failed to display this inhibition (Fig. 3a, b) presumably due to the loss of endogenous negative feedback.

Consistent with this, following WN1 stress, the cumulative integrated fluorescence started to decline in vehicle-treated mice (Fig. 3d). We observed that tonic CRH neuron activity during the white noise interval was either the same or below baseline levels in all vehicle-treated mice. However, in metyrapone-treated mice lacking the ability to synthesize CORT de novo, tonic CRH neural activity during this period was either the same or above baseline 

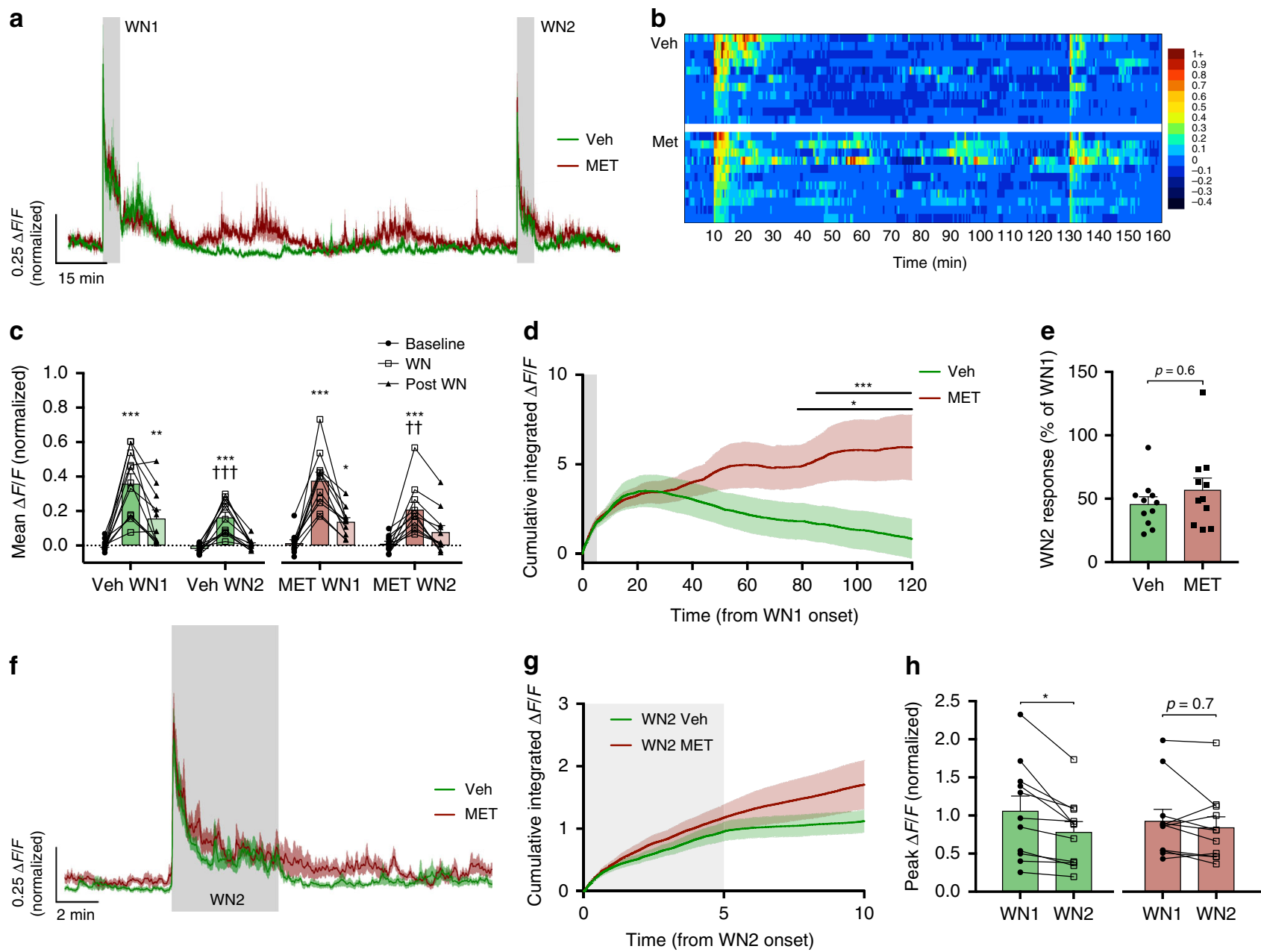

i
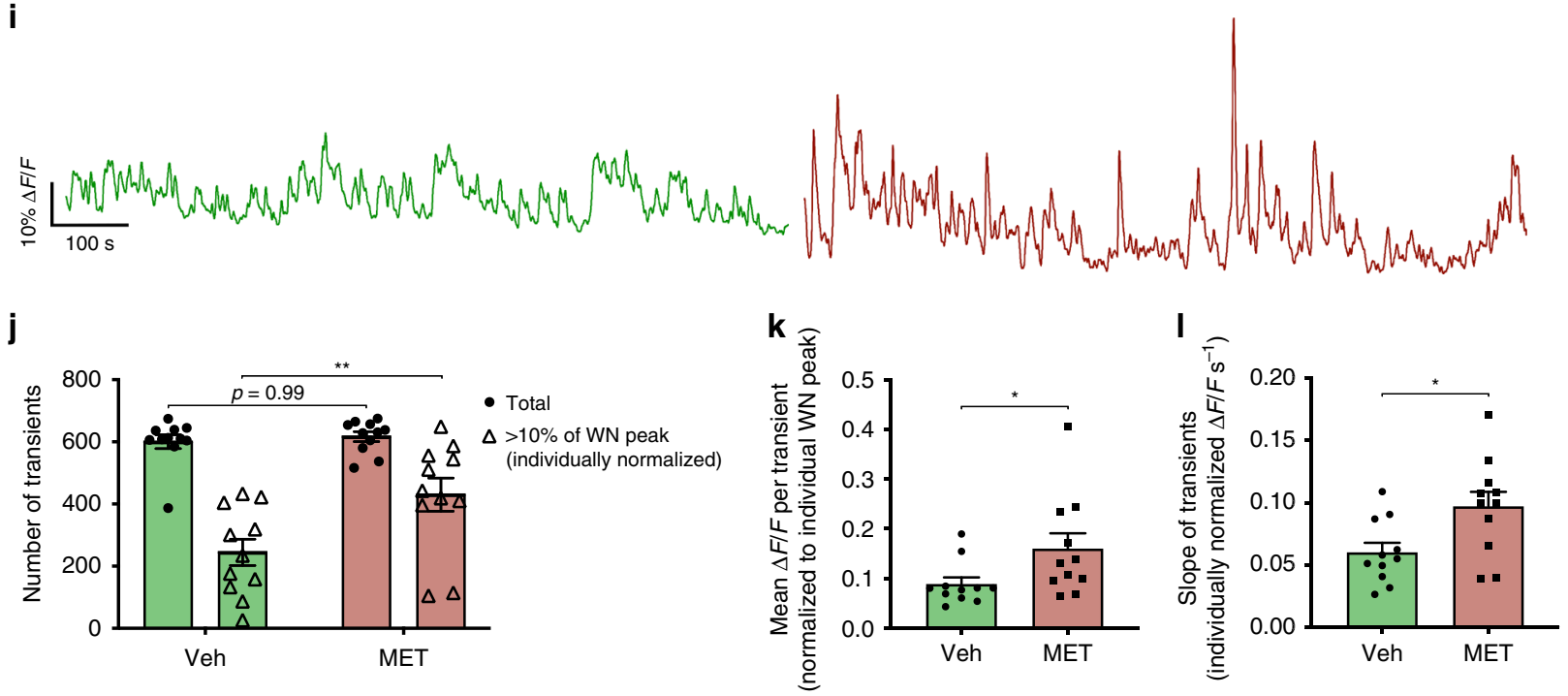

levels. Overall in the metyrapone-treated mice, the mean cumulative CRH neural activity remained elevated (Veh vs. MET cumulative $\Delta F / F, p<0.05$ from $78 \mathrm{~min}$ post $\mathrm{WN} 1$ onset, RM two-way ANOVA; Fig. 3d).

Despite these differences in tonic activity, a strong adaptive suppression of CRH neuron activity was observed in both groups in response to $\mathrm{WN} 2$ (mean $\mathrm{CRH}$ activity during $5 \mathrm{~min} \mathrm{WN2}$ : Veh-WN2 $0.16 \pm 0.03 \Delta F / F$ vs. MET-WN2 $0.21 \pm 0.04 \Delta F / F, p=$
0.98 RM two-way ANOVA; Fig. 3c) and the degree of suppression observed during WN2 was not different between the two conditions (Veh-WN2 $45.9 \pm 5.6 \%$ of WN1 response vs. MET-WN2 $57.1 \pm 9.3 \%$ of WN1, $p=0.4$ Mann-Whitney test; Fig. 3e). There was also no significant difference in the cumulative integrated fluorescence response to WN2 between groups $(p=$ 0.44 at 10 min from WN2 onset, RM two-way ANOVA; Fig. 3g). Interestingly, while vehicle-treated mice again showed a 
Fig. 3 CORT feedback slowly inhibits tonic CRH neuron excitability without affecting habituation. a Mean photometry signals of CRH neuron activity from two sequential WN stressors 120 min apart in vehicle and metyrapone-treated mice. $\mathbf{b}$ Heatmap of mean CRH neuron activity from all individual mice in 30 s bins. c Average $\Delta F / F$ across 5 min of $C R H$ neuron activity before, during, and after each $W N ; n=11$ per group, RM two-way ANOVA, ${ }^{*}<<0.05$ vs. baseline, $\uparrow p<0.05$ vs. respective WN1 timepoint, Holm-Sidak; ANOVA interaction $p=0.71$, ANOVA main effect of group $p=0.39$. d Cumulative integrated $\Delta F / F$ from the point of WN1 onset; RM two-way ANOVA, ${ }^{\star} p<0.05$ Veh vs. MET, Holm-Sidak; ANOVA interaction $p<0.001$, ANOVA main effect of group $p=0.19$. Lines indicate points at which statistical significance was reached and its duration. Gray shaded area indicates duration of WN. e CRH neuron activity during WN2 is shown as a percentage relative to WN1; Veh vs. MET, Mann-Whitney test. $\mathbf{f}$ Averaged photometry recordings of CRH neuron activity from vehicle- and metyrapone-treated mice showing the response to white noise 2 (WN2). $\mathbf{g}$ Cumulative integrated $\triangle F / F$ from the time of WN2 onset; RM two-way ANOVA, Veh vs. MET, Holm-Sidak; ANOVA interaction $p<0.001$, ANOVA main effect of group $p=0.38$. Gray shaded area indicates duration of WN. h Peak CRH $\Delta F / F$ at WN onset; RM two-way ANOVA, ${ }^{*} p<0.05$ vs. WN1, Holm-Sidak; ANOVA interaction $p=0.08$, ANOVA main effect of group $p=0.87$. i Representative photometry traces of tonic activity (during a period between WN1 and WN2) in individual vehicle- (left) or metyrapone- (right) treated mice. $\mathbf{j}$ Total number of GCaMP transients and proportion of transients larger than $10 \%$ of individual peak WN $\Delta F / F$; ${ }^{*} p<0.05$, one-way ANOVA, Tukey. k Average $\Delta F / F$ ( $\%$ of individual WN peak) of detected GCaMP transients during post-stress activity; ${ }^{*} p<0.05$, Mann-Whitney test. I Average slope (measured by amplitude/time) of detected GCaMP transients $\left(\Delta F / F\right.$ measured as $\%$ of individual $W N$ peak); ${ }^{*} p<0.05$, Mann-Whitney test. All data presented as mean $\pm \mathrm{SEM},{ }^{\star} / \mathrm{t} p<0.05,{ }^{\star \star} / \dagger p<0.01,{ }^{\star \star \star} / \mathrm{H \dagger} p<0.001$.

significant reduction in the peak response to WN2 (WN1 $1.0 \pm$ $0.19 \Delta F / F$ vs. WN2 $0.78 \pm 0.14 \Delta F / F ; p=0.02$ RM two-way ANOVA; Fig. $3 \mathrm{~h})$, this was not observed in metyrapone-treated mice (WN1 $0.93 \pm 0.15 \Delta F / F$ vs. WN2 $0.84 \pm 0.14 \Delta F / F ; p=0.7$ RM two-way ANOVA; Fig. 3h).

Given the significant effects of CORT feedback on tonic CRH activity, we decided to analyze the calcium transients during the inter-stress interval (from $15 \mathrm{~min}$ post-WN1, $100 \mathrm{~min}$ analysis; Fig. 3i). While the total number of GCaMP6s calcium events were not different between groups ( $p=0.99$ one-way ANOVA; Fig. $3 \mathrm{j}$ ), metyrapone-treated mice displayed an increased proportion of larger $(>10 \%$ of individual WN1 peak values) fluorescent transients $(p=0.005$ one-way ANOVA; Fig. $3 \mathrm{j})$. The overall mean amplitude of transients was also higher in metyraponetreated mice ( $p=0.03$ Mann-Whitney $t$ test; Fig. 3k). Interestingly, we also observed significantly faster rise times for fluorescent transients in the metyrapone group $(p=0.03$ Mann-Whitney $t$ test; Fig. 31). Therefore, the apparent inhibitory effects of CORT feedback are likely caused by reductions in event amplitudes, but not total event frequency, driving an offset in GCaMP6s fluorescence during tonic activity. These differences in tonic calcium events cannot be explained by differences in overall GCaMP fluorescence as there was no significant difference in the peak WN1 response (Fig. $3 \mathrm{~h}$ ) and mean responses to WN1 stress between groups (Fig. 3c).

Despite the significant CORT inhibition of tonic $\mathrm{CRH}$ activity, these results indicate that CORT is not involved in adaptive suppression of stress-evoked responses. Instead, past experience alone appeared to be sufficient to induce adaptation. Based on this observation, we theorized that CORT feedback preferentially modulates tonic CRH neuron activity, whereas adaptive changes to stress-evoked CRH neuron drive is experience gated.

CORT slowly inhibits tonic, but not stress-induced activity. We next tested whether exogenous CORT could inhibit stress-evoked $\mathrm{CRH}$ neural activity in response to a novel stressor. Previous work has consistently shown that exogenous CORT induces a strong suppression in stress-induced endocrine responses ${ }^{24,28-31}$, which has often been attributed to inhibition of CRH neuron activity $16,28,31$.

All mice were treated with metyrapone $90 \mathrm{~min}$ prior to experimental manipulation to block endogenous CORT synthesis. A subsequent single intraperitoneal (i.p.) injection of CORT (11ß,21-dihydroxy-4-pregnene-3,20-dione; $0.5 \mathrm{mg} / \mathrm{kg}$ in $0.86 \%$ dimethyl sulfoxide (DMSO)) rapidly induced high, physiological levels of circulating CORT, whereas vehicle-injected mice showed no change (Supplementary Fig. 3A). Following an initial response to the handling and injection stress, $\mathrm{CRH}$ neuron activity rapidly returned to baseline levels (Fig. $4 \mathrm{a}, \mathrm{b}$ ). We continued to record the tonic $\mathrm{CRH}$ neuron activity in the absence of external stress and observed a slow inhibition of tonic activity in the CORT treatment group $(-0.08 \pm 0.01 \Delta F / F$ from baseline, $85-125 \mathrm{~min}$ post injection, $n=12, p<0.0001$ RM two-way ANOVA; Fig. $4 \mathrm{c}$ ). Vehicle-treated controls did not exhibit the same slow inhibition, but instead sustained a stable level of tonic CRH activity $(0.004 \pm$ $0.01 \Delta F / F$ from baseline, $85-125$ min post injection, $n=12, p=$ 0.98 RM two-way ANOVA; Fig. 4c). The time course of the CORT inhibitory effect was most apparent when the cumulative integrated $\Delta F / F$ was plotted (Fig. $4 \mathrm{~d}$ ). This revealed that while the baseline activity between the vehicle and CORT groups started to diverge $\sim 40$ min following injection, a significant difference was not observed until $80 \mathrm{~min}$ (Veh vs. CORT cumulative $\Delta F / F, p<$ 0.05 from 80 min post injection, RM two-way ANOVA; Fig. 4d).

To test the impact of CORT feedback on novel stress-evoked $\mathrm{CRH}$ neuron responses, we next applied a white noise stress either $30 \mathrm{~min}$ ( $n=12$, Fig. $5 \mathrm{a}$, b) or $150 \mathrm{~min}(n=8$; Fig. 4 e) from the time of injection. These time points were chosen based on the kinetics of the inhibition by CORT feedback on tonic $\mathrm{CRH}$ neuron activity. The separation of baseline activity is not discernible during the initial $30 \mathrm{~min}$, but persists long after the injection. These time points also correlate to the fast nongenomic and slow genomic time windows of CORT actions ${ }^{32}$.

When a novel white noise stress was applied $150 \mathrm{~min}$ post injection ( $n=8$; Fig. 4 e), CORT treatment seemingly suppressed $\mathrm{CRH}$ neuron activity (mean activity during $5 \mathrm{~min}$ WN: Veh 0.39 $\pm 0.08 \Delta F / F$ vs. CORT $0.23 \pm 0.03 \Delta F / F, p=0.03$ RM two-way ANOVA; Fig. 4f). This inhibition appeared to result from the offset in baseline fluorescence induced by negative feedback rather than a change in the magnitude of the stress response itself. To determine if this was the case, we normalized the baseline to $10 \mathrm{~min}$ prior to the white noise to isolate the magnitude of the stress response (Fig. 4h). This revealed negligible differences in the $\mathrm{CRH}$ neuron response to white noise between groups (mean activity during 5 min WN: Veh $0.37 \pm 0.09 \Delta F / F$ vs. CORT $0.29 \pm$ $0.04 \Delta F / F, p=0.6 \mathrm{RM}$ two-way ANOVA; Fig. $4 \mathrm{i})$.

Responses to a novel white noise stress $30 \mathrm{~min}$ post injection $(n=12$, Fig. $5 \mathrm{a}, \mathrm{b})$ were also not different between vehicle and CORT-injected groups (mean activity during $5 \mathrm{~min} W \mathrm{~W}$; Veh $0.42 \pm 0.07 \Delta F / F$ vs. CORT $0.29 \pm 0.05 \Delta F / F, p=0.16 \mathrm{RM}$ twoway ANOVA; Fig. 5c). Furthermore, peak response (Veh $1.0 \pm$ $0.14 \Delta F / F$ vs. CORT $0.9 \pm 0.09 \Delta F / F, p=0.6$ Mann-Whitney test; Fig. $5 \mathrm{~d}$ ) and post white noise activity (Veh $0.11 \pm 0.03 \Delta F / F$ vs. CORT $0.03 \pm 0.04 \Delta F / F, p=0.4$ RM two-way ANOVA; Fig. $5 c$ ) were also unaffected by CORT at the 30 min time point. However, when the cumulative integrated $\triangle F / F$ was compared between vehicle and CORT groups, a small suppression of activity in the CORT condition was discernible (Fig. 5e), consistent with our 
a
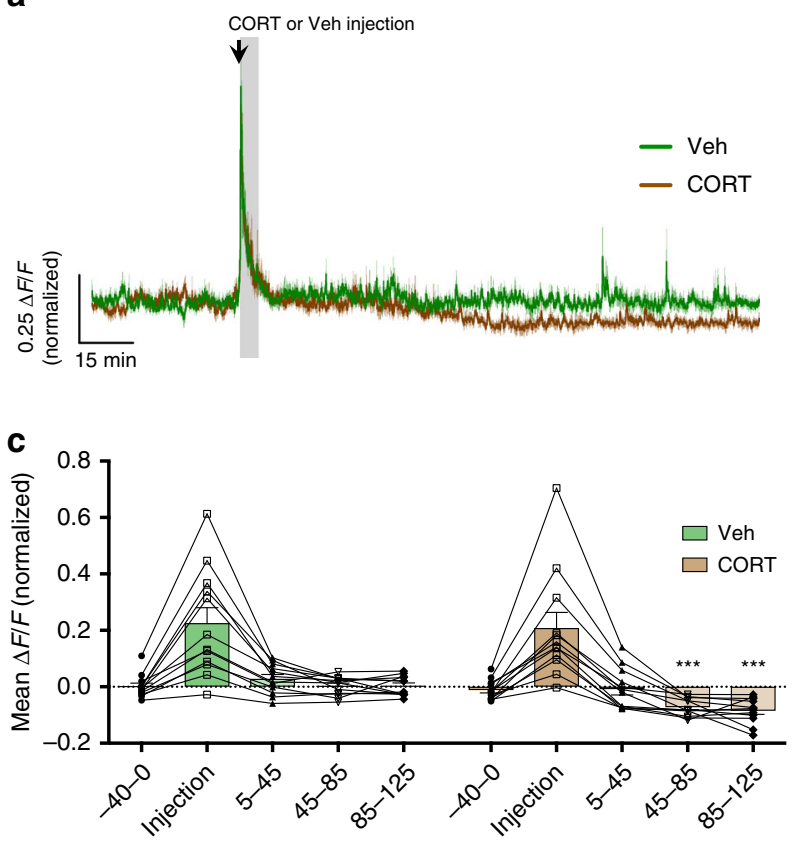

Time from injection (min)

e

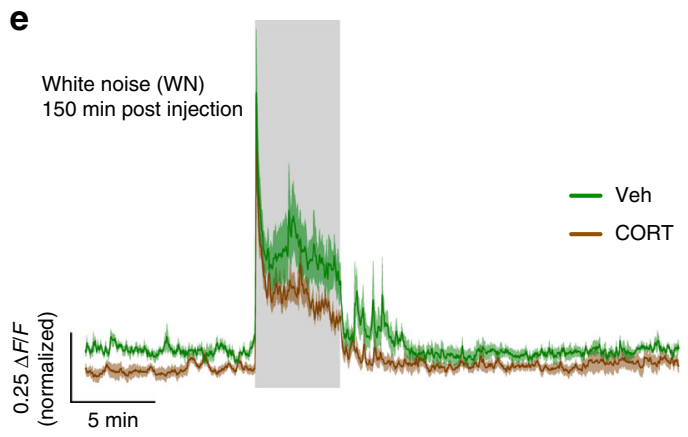

h

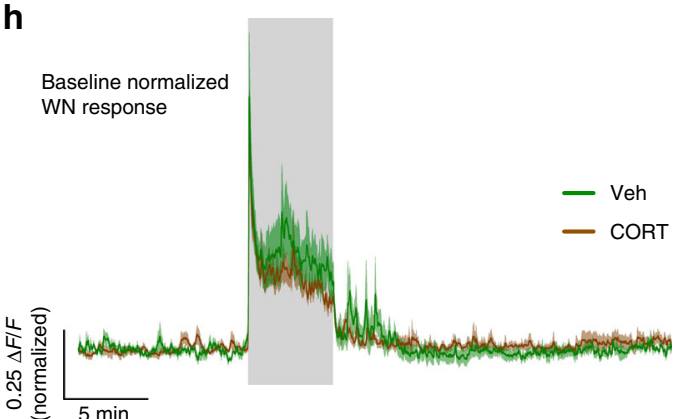

\section{b}

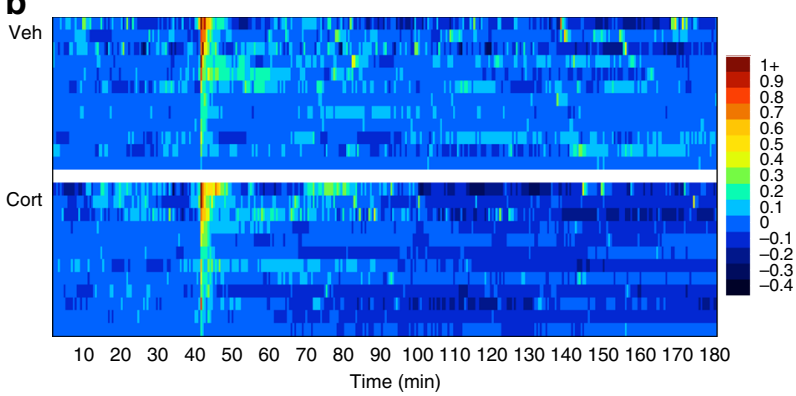

d

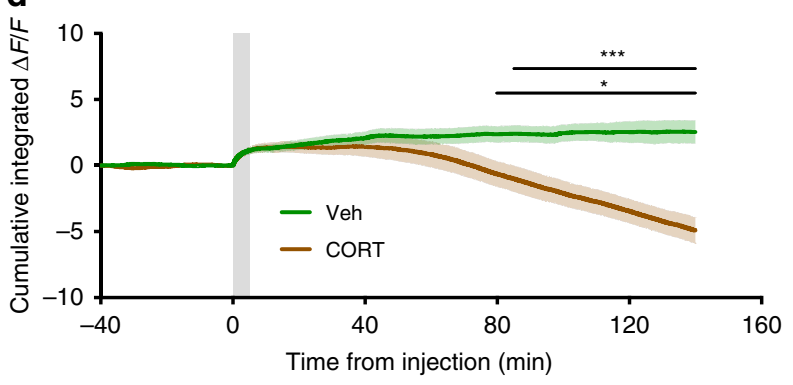

f

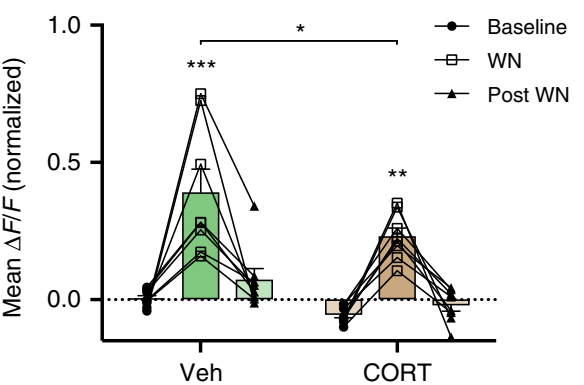

i

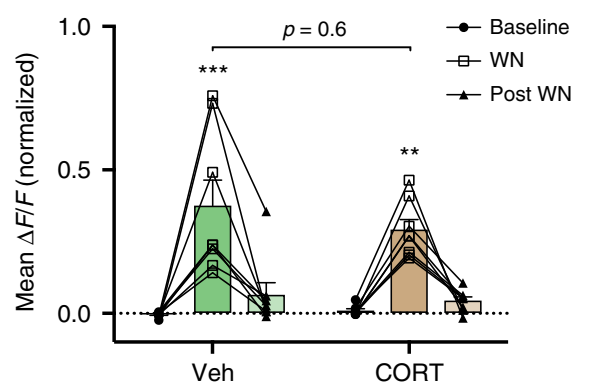

g

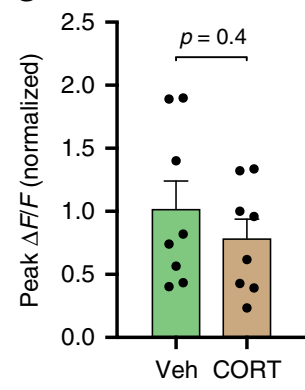

j

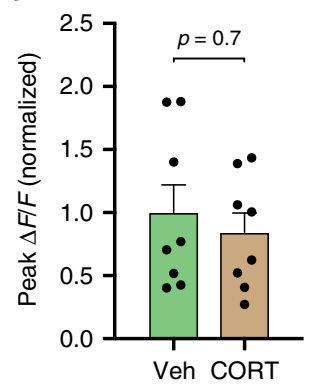

Fig. 4 CORT-negative feedback slowly suppresses basal but not stress-evoked CRH neuron activity. a Mean photometry signals of CRH neuron activity from vehicle- and CORT-treated mice. Gray shaded area indicates time of injection where handling/injection stress response is evident. $\mathbf{b}$ Heatmap of mean $\mathrm{CRH}$ neuron activity from all individual mice in $30 \mathrm{~s}$ bins. c Mean $\Delta F / F$ changes in 40 min bins; $n=12$ per group, RM two-way ANOVA, ${ }^{\star \star \star} p<0.001$ vs. baseline, Holm-Sidak; ANOVA interaction $p<0.001$, ANOVA main effect of group $p<0.001$. CRH neuron activity during injection stress (5 min bin) was not included in the statistical analysis. d Cumulative integrated $\Delta F / F$ of tonic $C R H$ neuron activity; RM two-way ANOVA, ${ }^{\star} p<0.05$ Veh vs. CORT, Holm-Sidak; ANOVA interaction $p<0.001$, ANOVA main effect of group $p=0.025$. Lines indicate points at which statistical significance was reached and its duration. Gray shaded area indicates stress response due to injection. e Averaged photometry recordings of the WN stress response 150 min after injection without baseline normalization. $\mathbf{f}$ Average $\triangle F / F$ across 5 min of $C R H$ neuron activity before, during, and after WN stress without baseline normalization; $n=8$ per group, RM two-way ANOVA, ${ }^{\star} p<0.05$ vs. baseline (unless otherwise indicated), Holm-Sidak; ANOVA interaction $p=0.45$, ANOVA main effect of group $p=0.01$. $g$ Peak $\Delta F / F$ at $W N$ onset without baseline normalization; Mann-Whitney test. $\mathbf{h}$ Averaged photometry recordings of the WN stress response $150 \mathrm{~min}$ after injection with baseline normalized to $10 \mathrm{~min}$ of activity prior to white noise. i Average $\Delta F / F$ across 5 min of $C R H$ neuron activity before, during, and after WN stress after baseline normalization; $n=8$ per group, RM two-way ANOVA, ${ }^{*}<0.05$ vs. Baseline, Holm-Sidak; ANOVA interaction $p=0.48$, ANOVA main effect of group $p=0.47$. j Peak $\Delta F / F$ at WN onset after baseline normalization; Mann-Whitney test. All data are presented as mean \pm SEM, ${ }^{\star} p<0.05,{ }^{\star \star} p<0.01,{ }^{\star \star \star} p<0.001$. 

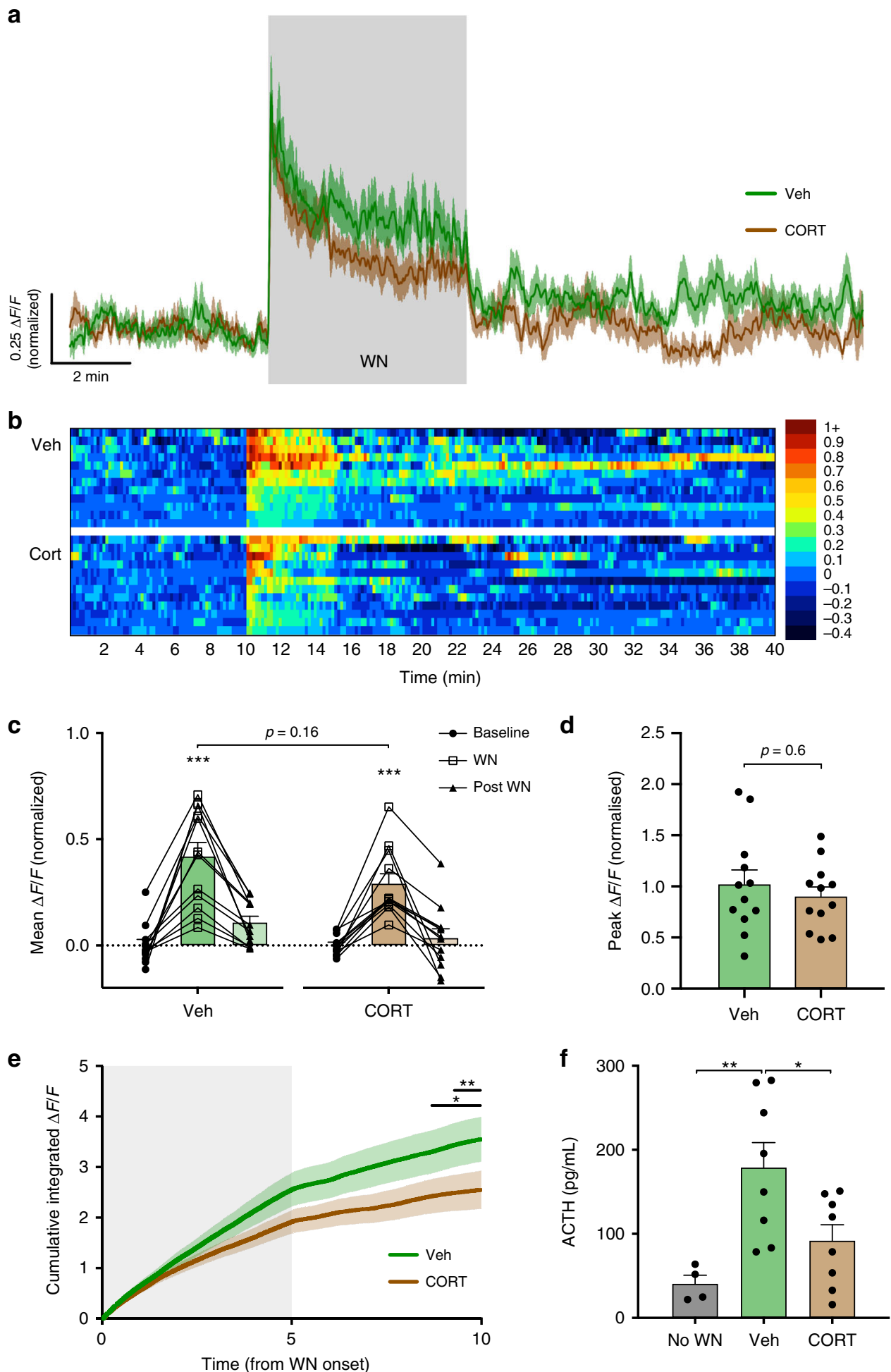

Fig. 5 Fast CORT feedback has little impact on CRH neural activity but faithfully suppresses ACTH release. a Mean photometry signals of CRH neuron activity during WN stress, 30 min after injection of either vehicle or CORT. $\mathbf{b}$ Heatmap of mean CRH neuron activity from all individual mice in $10 \mathrm{~s}$ bins. c Average $\Delta F / F$ across 5 min of $C R H$ neuron activity before, during, and after $W N ; n=12$ per group, RM two-way ANOVA, ${ }^{\star \star \star} p<0.001$ vs. baseline, Holm-Sidak; ANOVA interaction $p=0.19$, ANOVA main effect of group $p=0.14$. d Peak $\triangle F / F$ at WN onset; Mann-Whitney test. e Cumulative integrated $\Delta F / F$ from the time of WN stress; RM two-way ANOVA, ${ }^{\star} p<0.05$ Veh vs. CORT, Holm-Sidak; ANOVA interaction $p<0.001$, ANOVA main effect of group $p=0.21$. Lines indicate points at which statistical significance was reached and its duration. Gray shaded area indicates duration of WN. $\mathbf{f}$ Plasma ACTH levels 5 min post WN; one-way ANOVA, Tukey's multiple comparison test. All data presented as mean $\pm \mathrm{SEM},{ }^{\star} p<0.05,{ }^{\star \star} p<0.01,{ }^{\star \star \star} p<0.001$. 
a
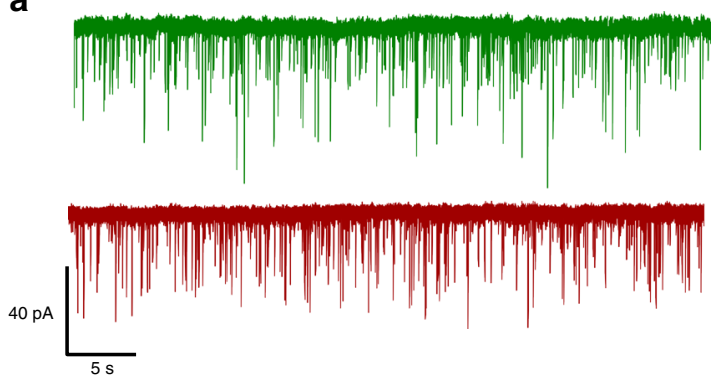

b

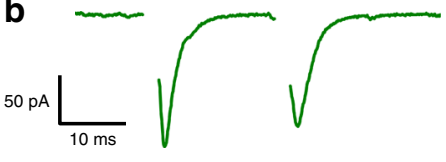

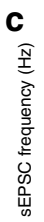

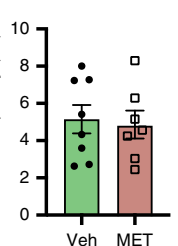

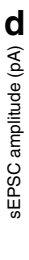

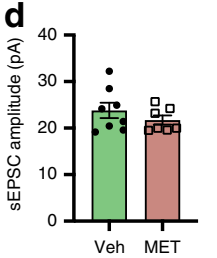

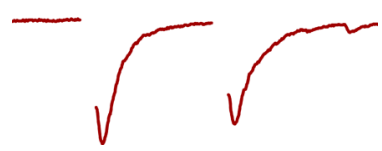

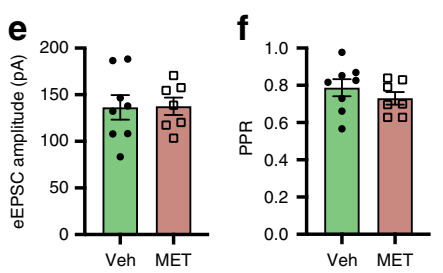

g

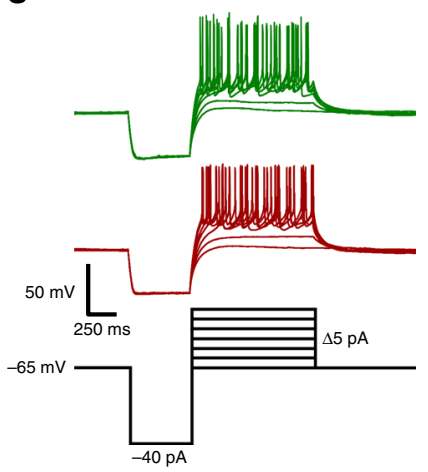

h
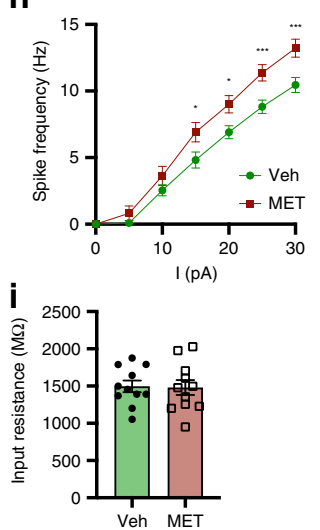

j

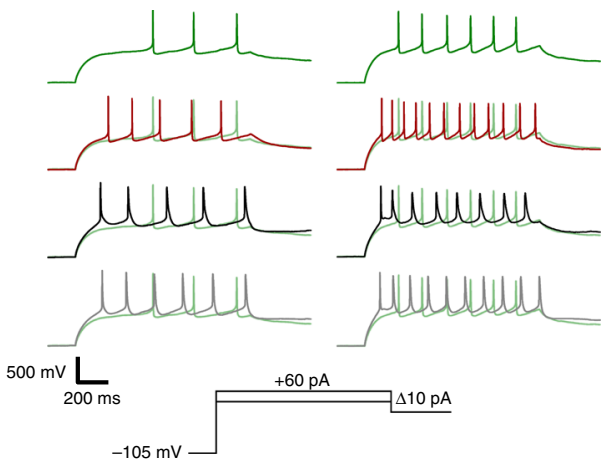

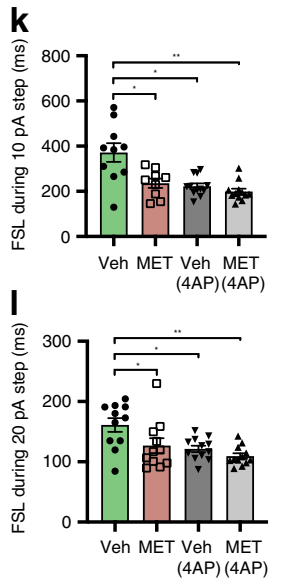

Fig. 6 CORT feedback increases first spike latency and decreases spike output. a Traces of sEPSC from individual CRH neurons from vehicle (green) or metyrapone- (red) treated mice. b Traces of paired-pulse eEPSC amplitudes from CRH neurons from vehicle (green) or metyrapone- (red) treated mice. c Mean sEPSC frequency; unpaired $t$ test, $p=0.8$. d Mean SEPSC amplitudes; unpaired $t$ test, $p=0.3$. e Mean eEPSC P1 amplitudes; unpaired $t$ test, $p=0.9$. f Paired pulse ratio (PPR); Mann-Whitney test, $p=0.3$. $\mathbf{g}$ Traces of CRH neuron spike output from vehicle (green) or metyrapone- (red) treated mice in response to varying current steps. $\mathbf{h}$ Spike frequency plotted for each 5 pA step; $n=11$ per group, RM two-way ANOVA, ${ }^{\star} p<0.05$ vs. vehicle, Holm-Sidak; ANOVA interaction $p=0.09$, ANOVA main effect of group $p<0.001$. i Input resistance from individual CRH neurons; unpaired $t$ test, $p=0.9$. $\mathbf{j}$ CRH neuron responses to $10 \mathrm{pA}$ (left) and $20 \mathrm{pA}$ (right) current steps with or without prior incubation with $4 \mathrm{AP}$ (Veh + 4AP in black, MET + 4AP in gray). k, I FSL during $10 \mathrm{pA}(\mathbf{k})$ and $20 \mathrm{pA}$ (I) steps from vehicle and metyrapone-treated mice with or without 4AP incubation. RM two-way ANOVA * $p<0.05$ Holm-Sidak; ANOVA interaction $p=0.003$, ANOVA main effect of group $p<0.001$. All data are presented as mean $\pm \mathrm{SEM},{ }^{\star} p<0.05,{ }^{\star \star} p<0.01$, ${ }^{\star \star \star} p<0.001$.

previous observations (Fig. 2h). Specifically, the cumulative integrated $\triangle F / F$ became significantly different between the vehicle and CORT groups $3.5 \mathrm{~min}$ following the termination of white noise (Fig. 5e).

We also assessed the effects of exogenous CORT on pituitary adrenocorticotropic hormone (ACTH) secretion. Control ACTH values obtained from metyrapone-treated mice without white noise were $40.6 \pm 10.3 \mathrm{pg} / \mathrm{mL}$ (no $\mathrm{WN}$; Fig. $5 f$ ). In response to white noise stress, ACTH levels from vehicle-treated mice were $178.8 \pm 29.7 \mathrm{pg} / \mathrm{mL}$ (measured $5 \mathrm{~min}$ after white noise stress). However, in mice previously treated with CORT, stress-evoked ACTH levels were $91.9 \pm 19.0 \mathrm{pg} / \mathrm{mL}$, which was significantly lower than the vehicle-treated group ( $p=0.04$, one-way ANOVA; Fig. 5f). These results show that fast CORT-negative feedback suppresses ACTH secretion while having a minor impact on $\mathrm{CRH}$ neuron activity.

Cellular correlates of CORT-negative feedback. The lack of CORT actions on stress-induced $\mathrm{CRH}$ neural activity in vivo challenges the long-standing textbook definitions of negative feedback. The most well-characterized fast, non-genomic effect of CORT on CRH neuron excitability is the suppression of spontaneous excitatory postsynaptic current (sEPSC) frequency ${ }^{16,33}$. Surprisingly, while it is generally accepted that CORT reduces $\mathrm{CRH}$ neuron spontaneous activity in acute brain slices, there is a lack of evidence to support an effect of CORT feedback on evoked CRH neuron activity.

Given the suppression of tonic CRH neuron excitability induced by CORT-negative feedback, we sought to identify potential plasticity mechanisms, which may underlie this. CrhIRES-Cre;Ai14 mice were treated with either vehicle or metyrapone and subsequently exposed to a single white noise stress as described above (Figs. 2 and 3). We then prepared acute brain slices containing the PVN 60 min after white noise stress and analyzed parameters of intrinsic and synaptic excitability using whole-cell patch-clamp electrophysiology.

No differences in sEPSC frequency or amplitude were observed between vehicle- and metyrapone-treated mice following white noise stress (mean sEPSC frequency: Veh $5.15 \pm 0.76 \mathrm{~Hz}, n=8$ vs. MET $4.86 \pm 0.75 \mathrm{~Hz}, n=7, p=0.79$ unpaired $t$ test; mean sEPSC amplitude: Veh $23.8 \pm 1.64 \mathrm{pA}, n=8$ vs. MET $21.7 \pm 1.0 \mathrm{pA}, p=$ 0.32 unpaired $t$ test; Fig. $6 \mathrm{a}, \mathrm{c}, \mathrm{d})$. Likewise, there were also no differences in evoked EPSC (eEPSC) amplitude or paired pulse ratio (PPR) between groups (mean eEPSC amplitude: Veh 136.4 $\pm 13.2 \mathrm{pA}, n=8$ vs. MET $137.5 \pm 9.3 \mathrm{pA}, n=7, p=0.95$ unpaired $t$ test; PPR: Veh $0.79 \pm 0.05, n=8$ vs. MET $0.73 \pm 0.03, n=7, p=$ 0.33 Mann-Whitney test; Fig. 6b, e, f). This lack of effect may be due to the fact that CORT will not remain elevated in brain slices maintained in vitro. Indeed, when CORT $(1 \mu \mathrm{M})$ was bath applied to brain slices from stress-naive mice, we observed a fast 
a
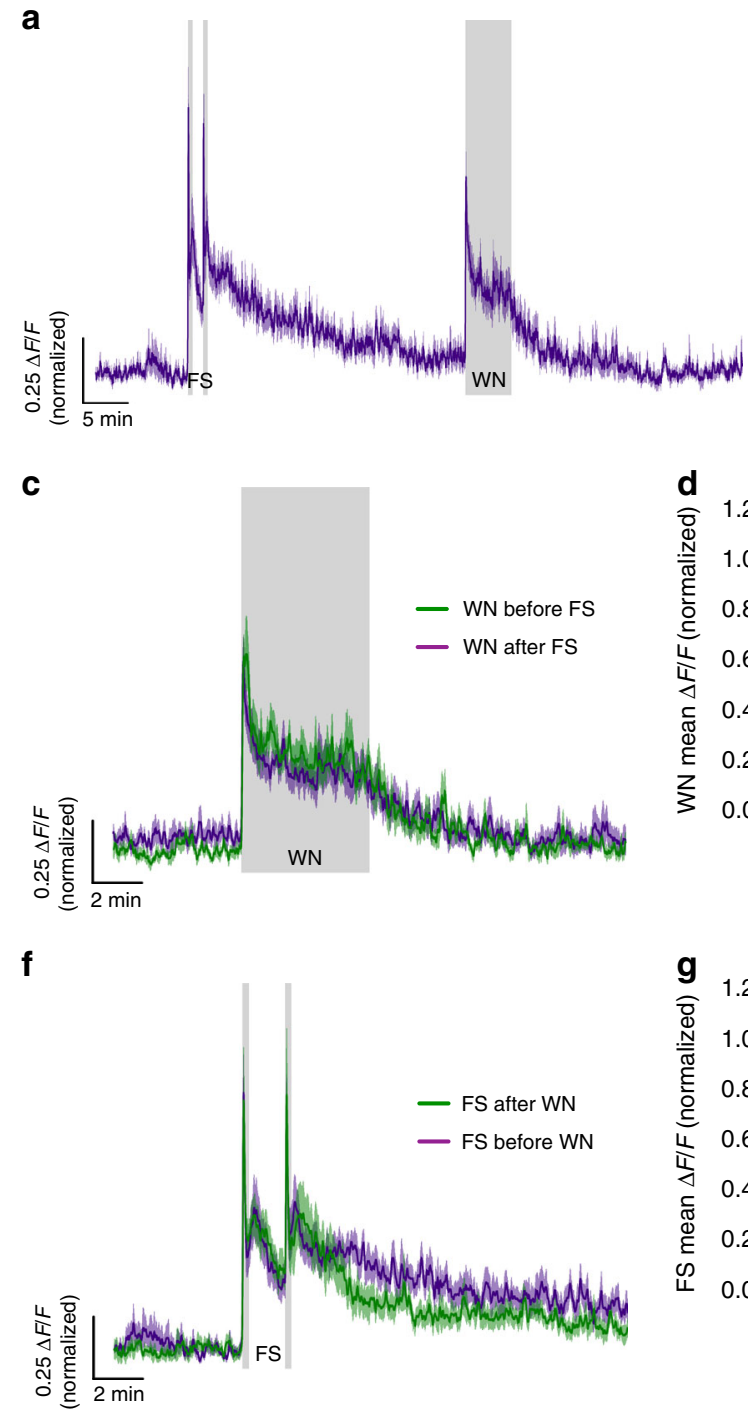

b
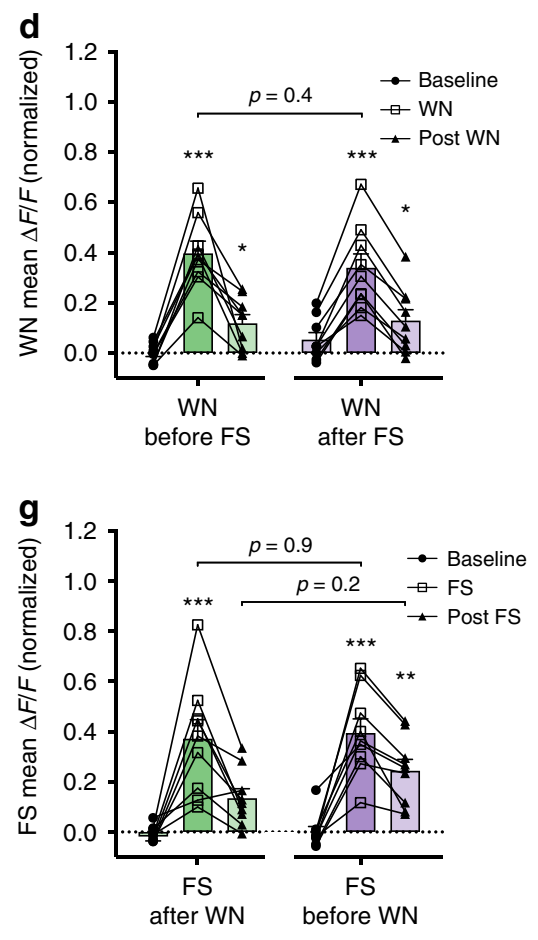
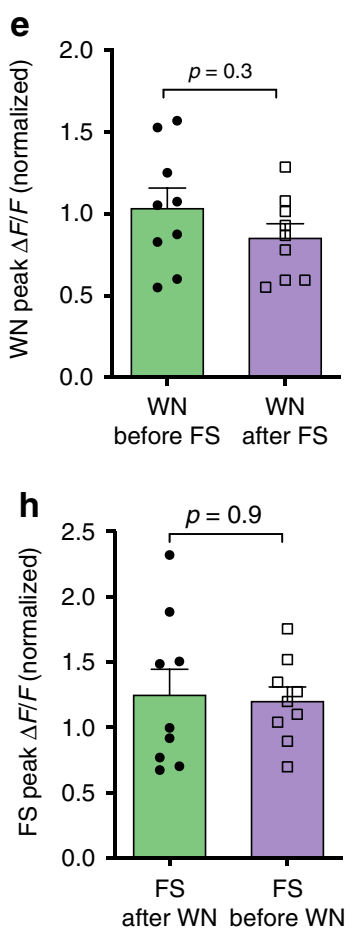

i

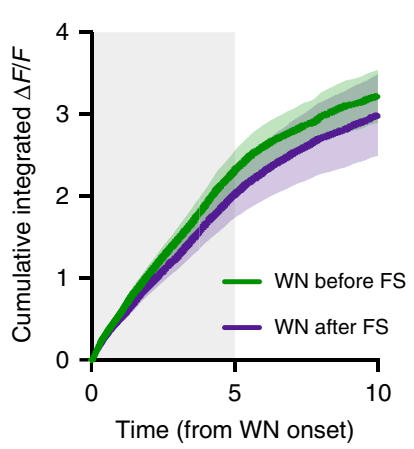

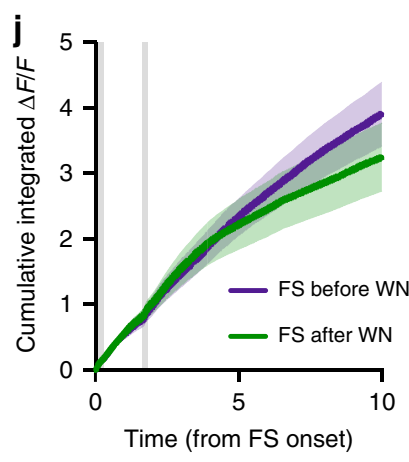

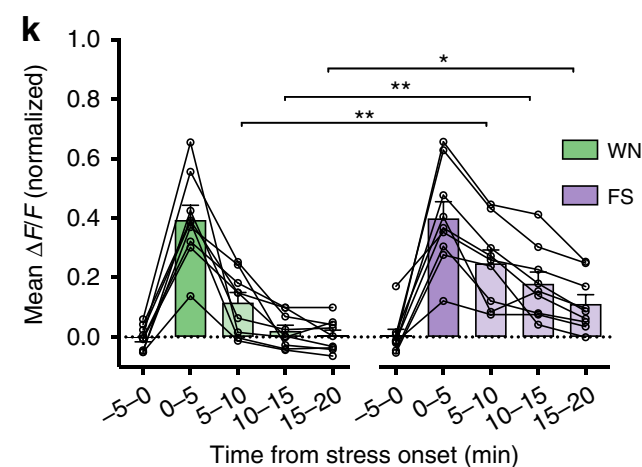

and significant reduction in sEPSC frequency recorded from CRH neurons (Supplementary Fig. 4A, B). However, bath applied CORT had no impact on eEPSC amplitude (Supplementary Fig. 4D, E) or PPR (Supplementary Fig. 4F). Likewise, bath applied CORT also failed to inhibit electrical stimulation induced elevations in excitability in GCaMP6s-expressing CRH neurons (Supplementary Fig. 4G, H).

We next examined whether in vivo CORT-negative feedback would alter the intrinsic excitability of $\mathrm{CRH}$ neurons in the presence of AMPA ((+/-)- $\alpha$-amino-3-hydroxy-5-methylisoxazole-4-propionic acid) and $\mathrm{GABA}_{\mathrm{A}}$ (gamma-aminobutyric acid [A]) receptor blockers. To measure intrinsic excitability in current clamp, CRH neurons were given a family of depolarizing current steps after a single $-40 \mathrm{pA}$ hyperpolarizing step (Fig. $6 \mathrm{~g}$ ). We found that $\mathrm{CRH}$ neurons from metyrapone-treated mice exposed to $\mathrm{WN}$ stress had higher intrinsic excitability and a greater spike output compared to vehicle controls $(p<0.05$ from 15 pA step onwards, $n=11$ (Veh) and $n=12$ (MET), RM twoway ANOVA; Fig. 6h). Input resistance was not different between groups (Veh $1.50 \pm 0.08 \mathrm{G} \Omega, n=11$ vs. MET $1.48 \pm 0.10 \mathrm{G} \Omega, n=$ $11, p=0.9$ unpaired $t$ test; Fig. 6i).

Previous work has suggested that CORT-negative feedback enhances a transient outward $\mathrm{K}^{+}$current in $\mathrm{CRH}$ neurons to regulate first spike latency (FSL) and excitability ${ }^{17}$. Consistent 
Fig. 7 Experience-gated habituation to white noise stress is dependent on stress familiarity. a Mean photometry signals of $\mathrm{CRH}$ neuron activity from mice receiving footshock (FS) followed by WN with a 30 min interval. b Mean photometry signals from mice receiving WN followed by FS. c Averaged photometry recordings of $\mathrm{CRH}$ neuron activity during WN stress when presented either first or second. d Average $\triangle F / F$ across 5 min of $C R H$ neuron activity before, during, and after each WN; $n=9$ per group, RM two-way ANOVA, ${ }^{\star} p<0.05$ vs. baseline, Holm-Sidak; ANOVA interaction $p=0.13$, ANOVA main effect of group $p=0.96$. e Peak $\Delta F / F$ response to WN stress presented either first or second; Mann-Whitney test. $\mathbf{f}$ Averaged photometry recordings of $\mathrm{CRH}$ neuron activity during footshock stress when presented either first or second. $\mathbf{g}$ Average $\Delta F / F$ across 5 min of $C R H$ neuron activity before, during, and after each FS; $n=9$ per group, RM two-way ANOVA, ${ }^{\star} p<0.05$ vs. baseline, Holm-Sidak; ANOVA interaction $p=0.44$, ANOVA main effect of group $p=0.31$. $\mathbf{h}$ Peak $\Delta F / F$ response to FS stress presented either first or second; Mann-Whitney test. $\mathbf{i}$ Cumulative integrated $\Delta F / F$ from the time of WN stress; RM two-way ANOVA, $p>0.99$ WN before FS vs. WN after FS at 10 min, Holm-Sidak; ANOVA interaction $p>0.99$, ANOVA main effect of group $p=0.52$. Gray shaded area indicates duration of WN. $\mathbf{j}$ Cumulative integrated $\triangle F / F$ from the time of FS stress; RM two-way ANOVA, $p>$ 0.99 FS before WN vs. FS after WN at $10 \mathrm{~min}$, Holm-Sidak; ANOVA interaction $p<0.001$, ANOVA main effect of group $p=0.67$. Gray shaded areas indicate timing of the two FSs ( $2 \mathrm{~s}$ duration each). $\mathbf{k}$ Mean $\Delta F / F$ response to the first presentations of WN or $F S$ stress in 5 min bins; $n=9$ per group, RM two-way ANOVA, ${ }^{*} p<0.05$ WN vs. FS, Holm-Sidak; ANOVA interaction $p=0.007$, ANOVA main effect of group $p=0.051$. All data presented as mean \pm SEM, ${ }^{\star} p<0.05,{ }^{\star \star} p<0.01,{ }^{\star \star \star} p<0.001$.

with this mechanism, $\mathrm{CRH}$ neurons from metyrapone-treated mice had a significantly shorter FSL compared to vehicle controls (FSL at 10 pA step: Veh $371.5 \pm 41.4 \mathrm{~ms}$ vs. MET $235.6 \pm 20.6 \mathrm{~ms}$, $p=0.02$ RM two-way ANOVA, $n=10$ (Veh) and $n=9$ (MET); Fig. 6k. FSL at $20 \mathrm{pA}$ step: Veh $161 \pm 11.4 \mathrm{~ms}$ vs. MET $126.8 \pm$ $12.4 \mathrm{~ms}, p=0.03 \mathrm{RM}$ two-way ANOVA, $n=11$ (Veh) and $n=$ 11 (MET); Fig. 61). To determine whether differences in FSL induced by negative feedback were mediated by an outward $\mathrm{K}^{+}$ conductance, we used 4-aminopyridine (4AP, $2 \mathrm{mM}$ ) to inhibit rapidly inactivating $\mathrm{K}^{+}$currents. FSL delays in $\mathrm{CRH}$ neurons from vehicle-treated mice following 4AP incubations were comparable to that of metyrapone-treated mice (FSL at $10 \mathrm{pA}$ step: Veh $+4 \mathrm{AP} 222.5 \pm 12.62 \mathrm{~ms}$ vs. MET $235.6 \pm 20.6 \mathrm{~ms}, p=$ 0.48 RM two-way ANOVA, $n=12(\mathrm{Veh}+4 \mathrm{AP})$ and $n=11$ (MET); Fig. $6 \mathrm{k}$. FSL at $20 \mathrm{pA}$ step: Veh $+4 \mathrm{AP} 121.4 \pm 5.3 \mathrm{~ms}$ vs. MET $126.8 \pm 12.4 \mathrm{~ms}, p=0.68 \mathrm{RM}$ two-way ANOVA, $n=12$ $(\mathrm{VEH}+4 \mathrm{AP})$ and $n=11$ (MET); Fig. 6l). Whereas 4AP had no significant effects on the FSL of CRH neurons from metyraponetreated mice (Fig. 6k, 1).

Overall, these data show that following stress, CORT-negative feedback suppresses intrinsic excitability and prolongs FSL. The timing of suppressed intrinsic excitability following stress matches the timing of inhibition of tonic CRH neuron activity measured with fiber photometry in vivo. Second, the faster and larger spontaneous calcium events observed in the metyrapone group in vivo (Fig. $3 \mathrm{j}-1$ ) are consistent with higher spiking excitability and shorter delay to spike observed in the metyrapone group in vitro.

Stress familiarity determines adaptation of CRH neuron output. Regardless of CORT feedback, adaptive stress habituation was consistently observed across the two sequential white noise epochs (Figs. 2 and 3). We proposed that this adaptation is experience gated, and therefore dependent on the familiarity of the stressor itself, but also requires regular exposure to the stress stimulus. To determine whether such adaptation was dependent on stress familiarity, we used a sequential stress paradigm with two different types of stressors: white noise and footshock (two shocks, $0.3 \mathrm{~mA}$, $2 \mathrm{~s}$ duration, $100 \mathrm{~s}$ interval) separated by $30 \mathrm{~min}$. Two groups of mice received white noise and footshock stressors in alternating order ( $n=9$; Fig. $7 \mathrm{a}, \mathrm{b})$. We speculated that adaptive habituation would not be observed with this paradigm due to the differing nature of the stressors. If this were the case, the white noise or footshock responses should be the same magnitude regardless of whether they were presented first or second.

CRH neural responses to white noise stress were near identical regardless of whether the white noise was presented first or 30 min following footshock (mean $\mathrm{CRH}$ activity during $5 \mathrm{~min} \mathrm{WN}$ : before FS, $0.40 \pm 0.05 \Delta F / F$ vs. after FS, $0.34 \pm 0.06 \Delta F / F, p=0.42$ RM two-way ANOVA; Fig. 7c, d). Footshock responses were also comparable between groups that either received it before or after white noise (mean CRH activity during $5 \mathrm{~min}$ from first FS onset: before WN, $0.40 \pm 0.06 \Delta F / F$ vs. after WN, $0.37 \pm 0.08 \Delta F / F, p=$ 0.91 RM two-way ANOVA; Fig. $7 \mathrm{f}-\mathrm{g}$ ). Peak responses to white noise or footshock (Fig. $7 \mathrm{e}, \mathrm{h}$ ), cumulative $\Delta F / F$ changes during and after white noise or footshock (Fig. 7i, j) were also unaffected by stress order.

To investigate the requirement for regular exposure to the stressor in maintaining long-term adaptation, mice received single daily exposure to white noise stress over 4 consecutive days (D1-4, Fig. 8a). Each exposure promoted adaptive habituation and subsequently suppressed the white noise response the next day. By day 4, white noise elicited merely a startle response, which rapidly returned to baseline levels (mean $\mathrm{CRH}$ activity during 5 min WN: day $10.47 \pm 0.08 \Delta F / F$ vs. day $20.30 \pm 0.04 \Delta F / F, p=$ 0.04 ; day 1 vs. day $30.22 \pm 0.06 \Delta F / F, p=0.002$; day 1 vs. day 4 $0.16 \pm 0.06 \Delta F / F, p<0.001$, RM two-way ANOVA; Fig 8c, d). While mean $\mathrm{CRH}$ neural activity during each white noise was consequently diminished (Fig. 8d), peak responses were unchanged, presumably due to a non-adaptive startle response (CRH peak response to $\mathrm{WN}$ day $11.0 \pm 0.14 \Delta F / F$ vs. day 40.78 $\pm 0.15 \Delta F / F, p=0.27$, RM two-way ANOVA; Fig. 8e).

This adaptive habituation to white noise stress could be extinguished following 3 weeks of white noise abstinence (round 2 day 1 mean $\mathrm{CRH}$ response during 5 min $\mathrm{WN}$ : $0.36 \pm 0.05 \Delta F / F$ vs. round 1 day $1, p=0.32$, RM two-way ANOVA; Fig. $8 \mathrm{~b}, \mathrm{~d}$ ), but could be relearnt, demonstrating the requirement for regular stress exposure to maintain the adaptive change. Blood CORT levels $30 \mathrm{~min}$ after each white noise significantly correlated with mean $\triangle F / F$ responses across the two 4 -day challenges (Fig. 8f; $r=0.9, p=0.003$, Pearson's correlation coefficient). Together, these data suggest that habituation of $\mathrm{CRH}$ neural activity is an important mechanism shaping long-term adaptation of the neuroendocrine stress response.

\section{Discussion}

There is consensus that CORT-negative feedback is essential for inhibiting the $\mathrm{CRH}$ neuron stress response. Despite the large body of evidence demonstrating fast and delayed forms of negative feedback on CRH neuron excitability in vitro $16,33,34$, how such mechanisms tune CRH neuron activity in vivo has until now been unknown. Using fiber photometry to observe the $\mathrm{CRH}$ neuron population activity in awake behaving mice, our findings reveal how this neural population responds to stress and the real-time dynamics of CORT-negative feedback. Specifically, we show that $\mathrm{CRH}$ neurons are tonically active in unstressed states and respond rapidly to sensory detection of external threats. We show that removal of the stressor alone is sufficient to initiate a fast decline in CRH activity, independent of CORT synthesis. This finding challenges the idea that CORT negative 
a
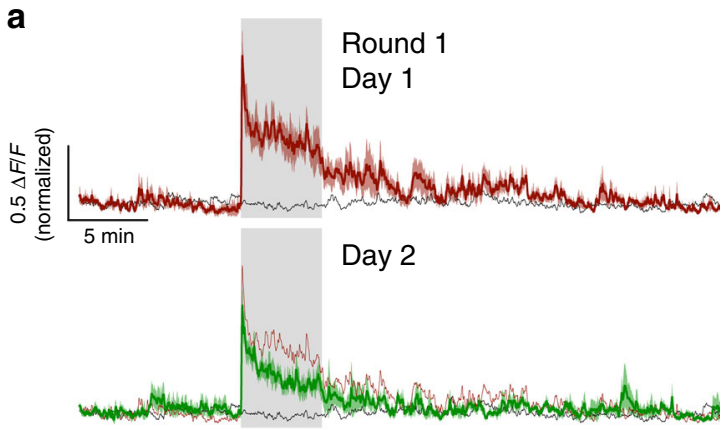

Day 3

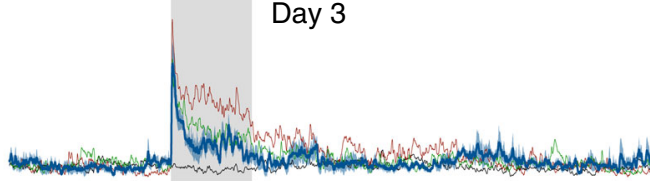

Day 4

C

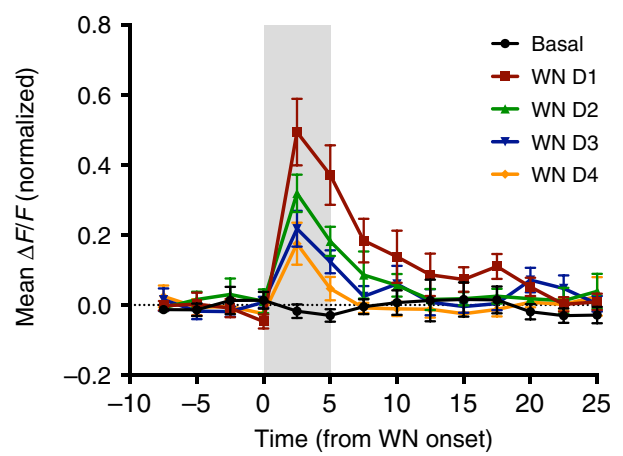

e

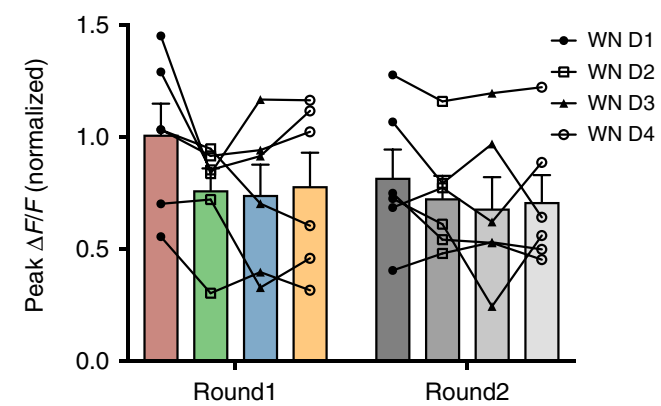

b

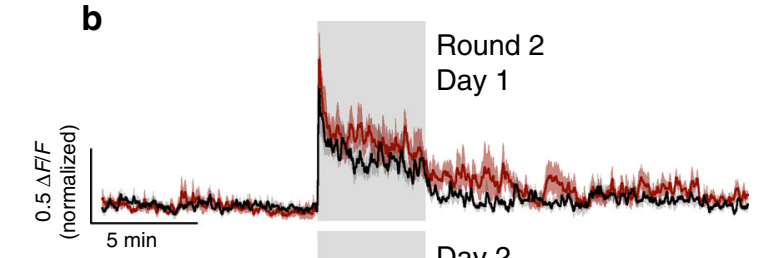

Day 2

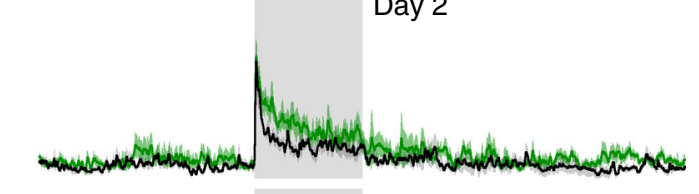

Day 3

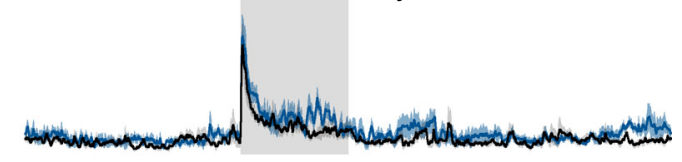

Day 4

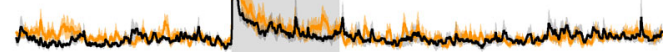

d
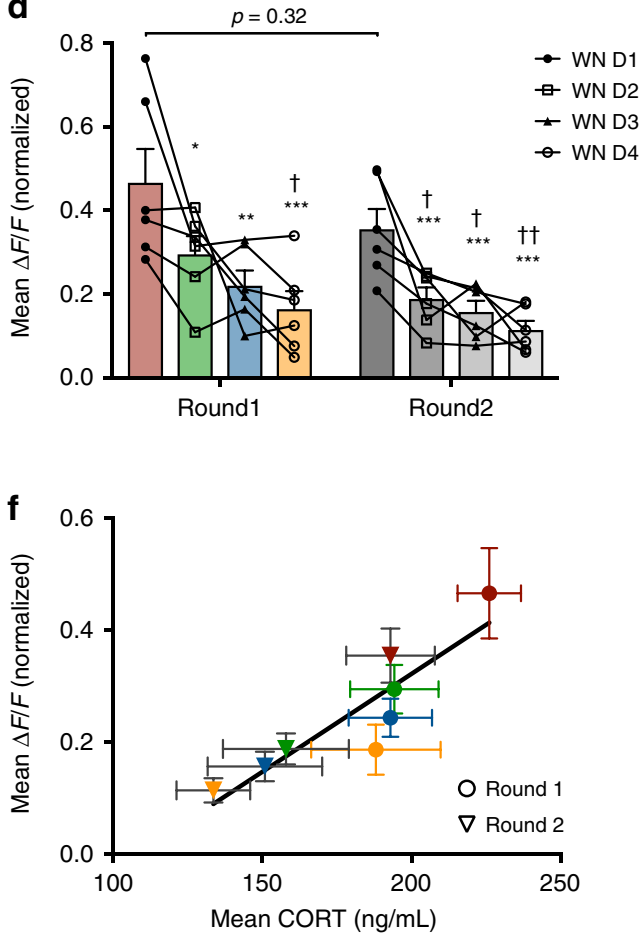

Fig. 8 Long-term adaptation of CRH neural activity to stress. a Mean photometry signals of CRH neuron activity from mice receiving daily WN stress over 4 days (round 1: day 1; red; day 2, green; day 3, blue; day 4, orange). Black trace indicates mean CRH neuron activity from the same cohort of mice on day 0 , in the absence of WN. b Mean photometry signals of CRH neuron activity from the same mice after a 3-week rest interval (with no stress) and then subsequently receiving daily WN stress over 4 more days (round 2: all recordings in black overlaid with corresponding round 1 WN response). c Mean $\Delta F / F$ changes in response to each $W N$ or no stress (black) in 2.5 min bins during round 1 . $\mathbf{d}$ Average $\Delta F / F$ of $C R H$ neuron activity during WN stress across the two rounds of repeated stress; $n=6$, RM two-way ANOVA, ${ }^{\star} p<0.05$ vs. round 1 WN day $1,{ }^{\dagger} p<0.05$ vs. round 2 WN day 1 , Holm-Sidak; ANOVA interaction $p=0.77$, ANOVA main effect of group $p=0.09$. e Peak $\Delta F / F$ at WN onset across the two rounds of repeated stress; RM two-way ANOVA, Holm-Sidak; ANOVA interaction $p=0.53$, ANOVA main effect of group $p=0.60$. $\mathbf{f}$ Correlation of mean CRH neuron activity during WN and corresponding post-stress blood CORT concentration across the two rounds (round 1 in circles and round 2 in triangles, 4 days of WN indicated by corresponding color; Pearson's $r=0.90, r^{2}=0.8$. All data presented as mean $\pm \mathrm{SEM},{ }^{\star} / \mathrm{t} p<0.05,{ }^{\star \star} / \mathrm{i} p<0.01,{ }^{\star \star \star} / \mathrm{it} p<0.001$. 
feedback induces a fast "shut off" of the neural stress response. While negative feedback is indeed essential for completely returning $\mathrm{CRH}$ neuron activity to the baseline state, this is a slow, gradual effect.

In both our exogenous and endogenous negative feedback models, we did not observe any substantial effect of CORT on stress-evoked CRH neural activity. Instead, experience-dependent habituation induced strong suppression of $\mathrm{CRH}$ neural responses to stress; whether the sequential white noise stressors were 30 min, $120 \mathrm{~min}$, or $24 \mathrm{~h}$ apart. These data show that CRH neuron responses are highly adaptive following repeated homotypic stress. This adaptation would act to prevent excessive stress responses against learnt non-harmful threats to limit unnecessary energy expenditure and maximize survival. Together, these findings reveal the importance of experience dependent plasticity in shaping neural responses to stress and redefine the principles of CORT negative feedback in the stress axis.

Past studies on CORT feedback have injected glucocorticoid receptor agonists and/or antagonists peripherally and observed suppression and enhancement in stress hormone secretion, respectively $14,24,28,29,31$. Our current work shows that fast CORT feedback suppresses stress-evoked ACTH release $30 \mathrm{~min}$ following injection. However, we observed only a subtle inhibition of stress-evoked CRH neuron activity during this period (Fig. 5). While we did not observe any changes in basal CRH neuron activity in the first $30 \mathrm{~min}$ following CORT injection (Fig. 4), we cannot rule out the possibility that CORT suppressed basal ACTH release during this period. Previous work has clearly shown that CORT negative feedback at the pituitary is important for the overall suppression of HPA axis output. In vitro studies in pituitary corticotroph cells have demonstrated that CORT can suppress electrical excitability, calcium elevations, and ACTH secretion within minutes ${ }^{35-37}$. CORT can also rapidly blunt $\mathrm{CRH}$-stimulated ACTH secretions in vivo ${ }^{38-40}$. While our results challenge long held beliefs regarding CORT actions on stress circuits in the brain, they are in fact consistent with past findings where exogenously applied glucocorticoids fail to impact c-fos expression in the PVN following stress ${ }^{28,41}$.

While CORT negative feedback did not substantially change stress-evoked $\mathrm{CRH}$ neuron responses, it was able to suppress tonic $\mathrm{CRH}$ neuron activity. This mechanism would act to reduce ongoing CRH secretion over extended time frames. We examined the cellular mechanisms which could underlie this CORTinduced, slow suppression of tonic activity. While fast CORT feedback is well known to inhibit spontaneous glutamatergic transmission ${ }^{16}$, we found no difference in spontaneous glutamate release following stress in the presence or absence of CORT negative feedback. Instead, stress induced CORT led to changes in intrinsic excitability. Negative feedback prolonged FSL, an effect that could be reversed with the potassium channel blocker 4AP. These findings are consistent with previous work showing that stress-induced CORT elevations reduce CRH neuron intrinsic excitability without affecting glutamate transmission ${ }^{17}$. These data also suggest that regulation of intrinsic excitability may be more important than inhibition of spontaneous glutamate transmission for the CORT induced suppression of tonic CRH neuron activity in vivo.

Our work has also identified that stress familiarity alone, independent of CORT negative feedback, is sufficient to strongly inhibit stress-evoked CRH neuron responses. Importantly, adaptive $\mathrm{CRH}$ neuron stress responses were not observed with unfamiliar, heterotypic stressors. Adaptive responses to white noise stress have previously been observed where CORT release is blunted following the second presentation of the same stressor ${ }^{26}$. Our observations suggest that habituation of peripheral stress hormone responses following homotypic stress are mediated by adaptation of CRH neural responses.

While the mean $\mathrm{CRH}$ neuron activity during white noise strongly habituated with repeated presentation of the familiar stress over 4 days, the peak response did not habituate. We speculate that the initial fast activation of $\mathrm{CRH}$ neurons is due to a startle response, which is important for quickly activating $\mathrm{CRH}$ neurons when exposed to an unexpected potential threat. Subsequently, as the animal has time to determine the nature of the threat, the stress response can then be adjusted to the level of danger. In the case of white noise, the animal learns over repeated presentations that the stimulus is non-harmful and therefore $\mathrm{CRH}$ neural activity returns towards baseline levels. This processing of "danger" information is likely mediated by upstream neural populations that are synaptically connected to $\mathrm{CRH}$ neurons. While the neural circuits involved in stress-specific habituation remains poorly understood, our work reinforces the importance of synaptic regulation of stress-evoked CRH neuron activity in addition to hormonal regulation.

While it may seem counter-intuitive, the overall lack of CORT inhibition of stress-evoked CRH neuron activity may be important for survival. Hypothalamic CRH neurons have recently been shown to serve critical roles in stress-induced behavior ${ }^{7}$, pheromone release $^{8}$, and encoding of valence ${ }^{9}$. Regardless of CORT milieu, appropriate behavioral responses in dangerous situations remain essential. Therefore, we argue that the lack of CORT effects on stress-evoked CRH neuronal activity may serve an important role in permitting normal neural responses and corresponding $\mathrm{CRH}-$ mediated stress behaviors, which facilitate survival.

In summary, we provide novel insight into how $\mathrm{CRH}$ neurons respond to stress in freely behaving mice. We have directly addressed the role of CORT-negative feedback on CRH activity for the first time and our results should prompt a reevaluation of the existing textbook definitions of negative feedback. Furthermore, we report that $\mathrm{CRH}$ neurons respond rapidly to sensory detection of threat and tune their output depending on stress familiarity. Thus, neural and endocrine mechanisms regulate different aspects of HPA axis function to shape an organism's responses to stress.

\section{Methods}

Animals. All mice were housed under a $12 \mathrm{~h}$ light/dark cycle in individually ventilated cages with ad libitum access to food and water. All experiments were conducted in accordance with the New Zealand Animal Welfare Act and approved by the University of Otago Animal Welfare and Ethics Committee.

Stereotaxic surgery. Adult (10-12-week-old) male Crh-IRES-Cre ${ }^{23}$ or Crh-IRES Cre;Ai14 (tdTomato reporter) mice ${ }^{42}$ were anesthetized with $2 \%$ isofluorane and placed in a stereotaxic frame. Adeno-associated virus (AAV) encoding GCaMP6s (AAV1.CAG.Flex.GCaMP6s.WPRZ.Sv40) or GFP (AAV9.Syn.DIO.EGFP.WPRE. hGH) was stereotaxically injected unilaterally into the PVN via a Hamilton syringe $(-0.8 \mathrm{~mm} \mathrm{AP},-0.25 \mathrm{~mm} \mathrm{ML},-4.5 \mathrm{~mm} \mathrm{DV})$ at a volume of $1 \mu \mathrm{L}$ over $10 \mathrm{~min}$. A fiberoptic cannula ( $400 \mu \mathrm{m}$ core, $0.48 \mathrm{NA}$; Doric Lenses) was then implanted at the same coordinates and secured using adhesive dental cement. All mice were given carprofen $(5 \mathrm{mg} / \mathrm{kg})$ and lidocaine $(2 \%)$ during surgery and allowed to recover for 4 weeks before experimental recordings.

Fiber photometry. Optical recordings of GCaMP6s fluorescence were acquired using a custom software acquisition system with optical components purchased from Doric Lenses $^{43}$. Excitation LEDs ( $465 \mathrm{~nm}$ blue and $405 \mathrm{~nm}$ violet) were sinusoidally modulated at 211 and $531 \mathrm{~Hz}$, respectively. Excitation wavelengths were relayed through a filtered fluorescence minicube (spectral bandwidth: 460-490 and $405 \mathrm{~nm}$ ) to a $400 \mu \mathrm{m} 0.48$ NA fiberoptic cable connected to the mouse. Light power for the $465 \mathrm{~nm}$ wavelength at the fiber tip was $35 \mu \mathrm{W}\left(70 \mu \mathrm{W} / \mathrm{mm}^{2}\right)$ and was estimated to drop off to $19 \mu \mathrm{W} / \mathrm{mm}^{2}$ within a distance of $0.2 \mathrm{~mm}$ from the fiber tip in brain tissue (61\% power attenuation). A single emission (filtered at $500-550 \mathrm{~nm}$ ) was detected using a femtowatt photoreceiver (2151, Newport) with a lensed fiber cable adapter. All signals were acquired at $10 \mathrm{kHz}$, digitized with a demodulation bandwidth of $\pm 5 \mathrm{~Hz}$, and down-sampled to a rate of $10 \mathrm{~Hz}$. 
Due to the duration of our recordings, a linear regression was used to correct for bleaching of the signal using the slope of the $405 \mathrm{~nm}$ signal fitted against the $465 \mathrm{~nm}$ signal, where $\Delta F / F=(465 \mathrm{~nm}-$ fitted 405$) /$ fitted 405 . We then normalized the $\Delta F / F$ for each experiment using the mean peak response to white noise onset from the vehicle control groups (Veh mean white noise peak $=1.0$ normalized $\Delta F / F$ ). Mice with peak response signals below $25 \% \Delta F / F$ were excluded from the study.

All experiments were conducted between zeitgeber time $0-5$ in the animal's home cage, which was placed in a custom-made apparatus $(40 \mathrm{~cm}$ length, $40 \mathrm{~cm}$ width, 40 $\mathrm{cm}$ height) with white walls and transparent lid. Speakers were mounted to the walls on two sides and a shock grid floor (Kinder Scientific) could be incorporated for induction of loud white noise $(85 \mathrm{~dB})$ or footshock $(0.3 \mathrm{~mA})$ stress without experimental handling. For experiments involving footshock, a custom bottomless cage was used in place of the home cage. Mice were habituated to the testing room and apparatus for 7 consecutive days prior to experimental manipulations.

CORT injection and white noise experiments: Mice were given an i.p. injection of metyrapone ( $100 \mu \mathrm{L}$ bolus i.p.; $75 \mathrm{mg} / \mathrm{kg}$; $25 \%$ PEG in saline) $90 \mathrm{~min}$ prior to the experiment. A 40-min baseline recording was taken prior to injection of CORT ( $100 \mu \mathrm{L}$ bolus i.p.; $0.5 \mathrm{mg} / \mathrm{kg} ; 0.84 \%$ DMSO in saline) or vehicle. This dose was chosen from a prior characterization experiment using wild-type C56BL6 mice where repeated tail blood samples were obtained (Supplementary Fig. 3A). This injection dose caused high but physiological blood concentrations of CORT comparable to an acute restraint stress response (Supplementary Fig. 3B). We have also previously observed such levels of CORT ${ }^{44}$ and other studies have also reported similar elevations in CORT levels following an acute restraint stress in mice, using the same enzyme-linked immunosorbent assay (ELISA) ${ }^{45,46}$. Two separate groups of mice were used to test white noise responses at either 30 or 150 min post injection. For each experiment, mice were randomly assigned to the vehicle or CORT group first and then received the alternative treatment 4 weeks later. Thus, each mouse served as its own internal control.

Sequential white noise experiments: All mice were given an i.p. injection of either metyrapone ( $100 \mu \mathrm{L}$ bolus i.p., $75 \mathrm{mg} / \mathrm{kg}$; $25 \%$ PEG in saline) or saline 90 min prior to the experiment. A $10 \mathrm{~min}$ baseline recording was taken prior to the onset of the first white noise. Two separate groups of mice were used to test two sequential white noise stress responses at either 30 or 120 min intervals. Each mouse served as their own internal control (metyrapone vs. vehicle) and repeated the experiment 4 weeks later, receiving the alternative treatment. For experiments involving daily white noise stressors, a single cohort of mice received four daily white noise stressors and repeated the daily stress protocol 3 weeks later.

White noise and footshock variable stress: Mice were presented with a footshock (two shocks separated by a 100 -s interval, $0.3 \mathrm{~mA}, 2 \mathrm{~s}$ duration) and white noise stress in alternating order with a 30-min interval between each stressor. Each mouse served as their own internal control (footshock then white noise vs. white noise then footshock) and repeated the experiment 4 weeks later, receiving the alternative stress order.

Brain slice electrophysiology and calcium imaging. Mice were euthanized via cervical dislocation and brains were sliced in an ice-cold cutting solution containing (in mM): $87 \mathrm{NaCl}, 2.5 \mathrm{KCl}, 25 \mathrm{NaHCO}_{3}, 1.25 \mathrm{NaH}_{2} \mathrm{PO}_{4}, 0.5 \mathrm{CaCl}_{2}, 6$ $\mathrm{MgCl}_{2}, 25 \mathrm{D}(+)$-glucose and 75 sucrose, saturated with $95 \% \mathrm{O}_{2} / 5 \% \mathrm{CO}_{2}$. Acute brain slices $(200 \mu \mathrm{m})$ containing the PVN were allowed to recover for at least $1 \mathrm{~h}$ in artificial cerebrospinal fluid (aCSF) consisting of (in $\mathrm{mM}$ ): $126 \mathrm{NaCl}, 2.5 \mathrm{KCl}, 26$ $\mathrm{NaHCO}_{3}, 1.25 \mathrm{NaH}_{2} \mathrm{PO}_{4}, 2.5 \mathrm{CaCl}_{2}, 1.5 \mathrm{MgCl}_{2}$ and $10 \mathrm{D}(+)$-glucose, saturated with $95 \% \mathrm{O}_{2} / 5 \% \mathrm{CO}_{2}$ at $30-32{ }^{\circ} \mathrm{C}$. All recordings were performed under an Olympus FV1000 confocal microscope at $30^{\circ} \mathrm{C}$ with a perfusion rate of $1-2 \mathrm{ml} /$ $\mathrm{min}$. CRH neurons were visualized by either tdTomato or GCaMP6s expression.

For voltage clamp loose patch recordings, borosilicate glass pipettes $(\sim 4 \mathrm{M} \Omega)$ were filled with aCSF and a low resistance seal $(\sim 10 \mathrm{M} \Omega)$ was achieved.

Noradrenaline $(50 \mu \mathrm{M})$ or $\mathrm{KCl}(7.5 \mathrm{mM})$ was applied to induce action potential firing and changes in GCaMP6s fluorescence were simultaneously imaged using a $488 \mathrm{~nm}$ Argon laser (Melles Griot).

For whole-cell recordings, glass pipettes were filled with an internal solution containing (in $\mathrm{mM}$ ): $120 \mathrm{~K}$-gluconate, $15 \mathrm{KCl}, 0.5 \mathrm{Na}_{2}$ EGTA, $2 \mathrm{Mg}_{2} \mathrm{ATP}, 0.4$ $\mathrm{Na}_{2} \mathrm{GTP}, 10 \mathrm{HEPES}$, and $5 \mathrm{Na}_{2}$-phosphocreatine (adjusted to $\mathrm{pH} 7.2$ with $\mathrm{KOH}$; adjusted to $290 \mathrm{mOsm}$ with sucrose). Neurons were voltage clamped at $-60 \mathrm{mV}$ to record EPSCs in the presence of picrotoxin $(50 \mu \mathrm{M})$. All current clamp intrinsic excitability experiments were performed with CNQX (cyanquixaline (6cyano-7-nitroquinoxaline-2,3-dione) $(10 \mu \mathrm{M})$ and picrotoxin $(50 \mu \mathrm{M})$. Each cell was held around $-65 \mathrm{mV}$ and we used a current step protocol to determine spike output and FSL. The step protocol consisted of a $0.5 \mathrm{~s}-40 \mathrm{pA}$ hyperpolarizing pulse, followed by increasing $1 \mathrm{~s}$ square steps from 0 to $+30 \mathrm{pA}$ in $5 \mathrm{pA}$ increments. Spikes were detected using a threshold search in Clampfit. FSL was calculated from the point of the depolarizing step initiation to the peak of the first spike.

Extracellular electrical stimulation was delivered using a monopolar glass electrode filled with aCSF. Biphasic paired pulse stimulations were delivered at $30-100 \mu \mathrm{A}$ at $20 \mathrm{~ms}$ intervals. Trains of electrical stimulations to evoke GCaMP6s responses were delivered at $100 \mu \mathrm{A}$ at $10 \mathrm{~Hz}$ for $5 \mathrm{~s}$.

Electrophysiological recordings were collected with a Multiclamp 700B amplifier (Molecular Devices), filtered at $2 \mathrm{kHz}$, and digitized using the Digidata 1440a (Molecular Devices). sEPSC currents were analyzed using Mini Analysis and all other electrophysiological data were analyzed with Clampfit 10 (Molecular Devices). GCaMP6s images were acquired using Fluoview 1000 at $\sim 2$ $\mathrm{Hz}$ frame rate and analyzed using Image J. Changes in fluorescence $(\Delta F / F)$ were calculated, where $F$ is the averaged baseline fluorescence for each region of interest.

Blood collection and ELISA. Tail blood samples were collected via heparinized capillary tubes. All mice were previously habituated to handling for at least 7 consecutive days. Plasma corticosterone was measured using an ELISA (Arbor Assays) according to the manufacturer's instructions. For ACTH measurements, mice were decapitated and trunk bloods were collected in lavender EGTA-coated tubes. All samples were kept on ice and centrifuged at $4{ }^{\circ} \mathrm{C}$ within minutes of collection. ACTH was measured using an ELISA (MD Bioproducts) according to the manufacturer's instructions.

Immunohistochemistry. GCaMP6s was labeled in fixed coronal brain sections (30 $\mu \mathrm{m}$ ) with a GFP antibody (chicken anti-GFP; 1:3000; Aves Labs) and visualized using Alexa Fluor 488 goat anti-chicken IgG (1:500; Molecular Probes, Life Technologies). Sections were imaged under confocal microscopy (Zeiss LSM 710) and analyzed using Image J to quantify GCaMP and CRH-tdTomato colocalization. We observed GCaMP6s transfection in $58.1 \pm 2.1 \%$ of CRH neurons in the ipsilateral PVN and $88.0 \pm 1.8 \%$ of GCaMP6s-transfected cells were positive for CRH-tdTomato (Fig. 1a and Supplementary Table 1).

Data analysis and statistics. Photometry data were processed using Prism and Excel to calculate linear regression and $\Delta F / F$ changes. We did not correct for any movement artifacts as they had minimal impact on the overall recording. Brain slice calcium images were acquired and analyzed using Fluoview 1000 and Image J, where regions of interest were drawn around each CRH neuron. For in vitro GCaMP6s imaging, corrections for photobleaching were not necessary and $\Delta F / F$ changes were normalized to the baseline.

For calculating correlations in spike to $\Delta F / F$ change in vitro, total spike counts were collected from each phasic burst-firing window using the pClamp 10 threshold search. Each phasic burst window was defined as a period where GCaMP6s fluorescence was elevated $(>10 \% \Delta F / F)$ and returned to baseline levels Total accumulation of GCaMP6s fluorescence during each burst was correlated with the spike count using Prism. Spontaneous synaptic currents and photometry GCaMP6s transients were detected using MiniAnalysis.

All data are presented as mean \pm SEM in the figures and text. All group comparisons for photometry data were performed using RM multiple comparisons two-way ANOVA (Holm-Sidak post hoc test), unless otherwise stated. All statistical analyzes were performed using Prism. ${ }^{\star} P<0.05,{ }^{* *} p<0.01$, and ${ }^{\star * *} p<0.001$.

Reporting summary. Further information on research design is available in the Nature Research Reporting Summary linked to this article.

\section{Data availability}

All datasets supporting the findings of this study are available upon reasonable request.

Received: 16 April 2019; Accepted: 11 November 2019; Published online: 13 December 2019

\section{References}

1. Selye, H. A syndrome produced by diverse nocuous agents. Nature 138, 32 (1936).

2. Herman, J. Neural control of chronic stress adaptation. Front. Behav. Neurosci. 7, 61 (2013).

3. McEwen, B. S. \& Liston, C. Mediators of Glucocorticoid-Regulated Adaptive Plasticity (Oxford University Press, 2017)

4. Li, Y. et al. Hypothalamic circuits for predation and evasion. Neuron 97, 911-924. e915 (2018)

5. Park, S.-G. et al. Medial preoptic circuit induces hunting-like actions to target objects and prey. Nat. Neurosci. 21, 364 (2018).

6. Kunwar, P. S. et al. Ventromedial hypothalamic neurons control a defensive emotion state. Elife 4, e06633 (2015).

7. Füzesi, T., Daviu, N., Wamsteeker Cusulin, J. I., Bonin, R. P. \& Bains, J. S. Hypothalamic CRH neurons orchestrate complex behaviours after stress. Nat. Commun. 7, 11937 (2016)

8. Sterley, T.-L. et al. Social transmission and buffering of synaptic changes after stress. Nat. Neurosci. 21, 393 (2018).

9. Kim, J. et al. Rapid, biphasic CRF neuronal responses encode positive and negative valence. Nat. Neurosci. 1, https://doi.org/10.1038/s41593-019-0342-2 (2019).

10. McEwen, B. S. Physiology and neurobiology of stress and adaptation: central role of the brain. Physiol. Rev. 87, 873-904 (2007). 
11. McCarty, R. Learning about stress: neural, endocrine and behavioral adaptations. Stress 19, 449-475 (2016).

12. Grissom, N. \& Bhatnagar, S. Habituation to repeated stress: get used to it. Neurobiol. Learn. Mem. 92, 215-224 (2009).

13. Bains, J. S., Cusulin, J. I. W. \& Inoue, W. Stress-related synaptic plasticity in the hypothalamus. Nat. Rev. Neurosci. 16, 377 (2015).

14. Tasker, J. G. \& Herman, J. P. Mechanisms of rapid glucocorticoid feedback inhibition of the hypothalamic-pituitary-adrenal axis. Stress 14, 398-406 (2011).

15. Herman, J. P. et al. Regulation of the hypothalamic-pituitary-adrenocortical stress response. Compr. Physiol. 6, 603 (2016).

16. Di, S., Malcher-Lopes, R., Halmos, K. C. \& Tasker, J. G. Nongenomic glucocorticoid inhibition via endocannabinoid release in the hypothalamus: a fast feedback mechanism. J. Neurosci. 23, 4850-4857 (2003).

17. Senst, L., Baimoukhametova, D., Sterley, T.-L. \& Bains, J. S. Sexually dimorphic neuronal responses to social isolation. Elife 5, e18726 (2016)

18. Wamsteeker Cusulin, J. I., Füzesi, T., Inoue, W. \& Bains, J. S. Glucocorticoid feedback uncovers retrograde opioid signaling at hypothalamic synapses. Nat. Neurosci. 16, 596 (2013)

19. Inoue, W. et al. Noradrenaline is a stress-associated metaplastic signal at GABA synapses. Nat. Neurosci. 16, 605 (2013).

20. Kuzmiski, J. B., Marty, V., Baimoukhametova, D. V. \& Bains, J. S. Stressinduced priming of glutamate synapses unmasks associative short-term plasticity. Nat. Neurosci. 13, 1257 (2010).

21. Cusulin, J. I. W., Senst, L., Teskey, G. C. \& Bains, J. S. Experience salience gates endocannabinoid signaling at hypothalamic synapses. J. Neurosci. 34, 6177-6181 (2014).

22. Kim, J. S. \& Iremonger, K. J. Temporally tuned corticosteroid feedback regulation of the stress axis. Trends in Endocrinol. Metab. 30, 783-792 (2019).

23. Taniguchi, H. et al. A resource of Cre driver lines for genetic targeting of GABAergic neurons in cerebral cortex. Neuron 71, 995-1013 (2011).

24. Andrews, M. H., Wood, S. A., Windle, R. J., Lightman, S. L. \& Ingram, C. D. Acute glucocorticoid administration rapidly suppresses basal and stress-induced hypothalamo-pituitary-adrenal axis activity. Endocrinology 153, 200-211 (2012).

25. Windle, R. et al. Endocrine and behavioural responses to noise stress: comparison of virgin and lactating female rats during non-disrupted maternal activity. J. Neuroendocrinol. 9, 407-414 (1997).

26. Atkinson, H., Wood, S., Kershaw, Y., Bate, E. \& Lightman, S. Diurnal variation in the responsiveness of the hypothalamic-pituitary-adrenal axis of the male rat to noise stress. J. Neuroendocrinol. 18, 526-533 (2006).

27. Yuan, Y. et al. Reward inhibits paraventricular CRH neurons to relieve stress. Curr. Biol. https://doi.org/10.1016/j.cub.2019.02.048 (2019).

28. Evanson, N. K., Tasker, J. G., Hill, M. N., Hillard, C. J. \& Herman, J. P. Fast feedback inhibition of the HPA axis by glucocorticoids is mediated by endocannabinoid signaling. Endocrinology 151, 4811-4819 (2010).

29. Osterlund, C. D. et al. Glucocorticoid fast feedback inhibition of stressinduced ACTH secretion in the male rat: rate independence and stress-state resistance. Endocrinology 157, 2785-2798 (2016).

30. Thrivikraman, K., Nemeroff, C. B. \& Plotsky, P. M. Sensitivity to glucocorticoid-mediated fast-feedback regulation of the hypothalamic-pituitary-adrenal axis is dependent upon stressor specific neurocircuitry. Brain Res. 870, 87-101 (2000).

31. Franco, A. J. et al. Sensitization of the hypothalamic-pituitary-adrenal axis in a male rat chronic stress model. Endocrinology 157, 2346-2355 (2016).

32. Joels, M. Corticosteroids and the brain. J. Endocrinol. 238, 121-130 (2018).

33. Wamsteeker, J. I., Kuzmiski, J. B. \& Bains, J. S. Repeated stress impairs endocannabinoid signaling in the paraventricular nucleus of the hypothalamus. J. Neurosci. 30, 11188-11196 (2010).

34. Bittar, T. P. et al. Corticosterone mediated functional and structural plasticity in corticotropin-releasing hormone neurons. Neuropharmacology. https://doi. org/10.1016/j.neuropharm.2019.1002.1017 (2019)

35. Deng, Q. et al. Rapid glucocorticoid feedback inhibition of ACTH secretion involves ligand-dependent membrane association of glucocorticoid receptors. Endocrinology 156, 3215-3227 (2015).

36. Duncan, P. J., Tabak, J., Ruth, P., Bertram, R. \& Shipston, M. J. Glucocorticoids inhibit CRH/AVP-evoked bursting activity of male murine anterior pituitary corticotrophs. Endocrinology 157, 3108-3121 (2016).

37. Romanò, N., McClafferty, H., Walker, J. J., Le Tissier, P. \& Shipston, M. J. Heterogeneity of calcium responses to secretagogues in corticotrophs from male rats. Endocrinology 158, 1849-1858 (2017).

38. Cole, M. A., Kim, P. J., Kalman, B. A. \& Spencer, R. L. Dexamethasone suppression of corticosteroid secretion: evaluation of the site of action by receptor measures and functional studies. Psychoneuroendocrinology 25, 151-167 (2000).

39. Hinz, B. \& Hirschelmann, R. Rapid non-genomic feedback effects of glucocorticoids on CRF-induced ACTH secretion in rats. Pharm. Res. 17, 1273-1277 (2000).
40. Russell, G. M. et al. Rapid glucocorticoid receptor-mediated inhibition of hypothalamic-pituitary-adrenal ultradian activity in healthy males. $J$. Neurosci. 30, 6106-6115 (2010).

41. Weiser, M. J., Osterlund, C. \& Spencer, R. L. Inhibitory effects of corticosterone in the hypothalamic paraventricular nucleus $(\mathrm{PVN})$ on stress-induced adrenocorticotrophic hormone secretion and gene expression in the PVN and anterior pituitary. J. Neuroendocrinol. 23, 1231-1240 (2011).

42. Wamsteeker Cusulin, J. I., Füzesi, T., Watts, A. G. \& Bains, J. S. Characterization of corticotropin-releasing hormone neurons in the paraventricular nucleus of the hypothalamus of Crh-IRES-Cre mutant mice. PLoS ONE 8, e64943 (2013).

43. Clarkson, J. et al. Definition of the hypothalamic GnRH pulse generator in mice. Proc. Natl Acad. Sci. USA 114, E10216-E10223 (2017).

44. Kim, J. S. et al. Anxiogenic and stressor effects of the hypothalamic neuropeptide RFRP-3 are overcome by the NPFFR antagonist GJ14. Endocrinology 156, 4152-4162 (2015).

45. Liu, Y. et al. SIRT3 mediates hippocampal synaptic adaptations to intermittent fasting and ameliorates deficits in APP mutant mice. Nat. Commun. 10, 1886 (2019).

46. Yang, J. A. et al. Acute psychosocial stress inhibits LH pulsatility and Kiss1 neuronal activation in female mice. Endocrinology 158, 3716-3723 (2017).

\section{Acknowledgements}

We are extremely grateful to Professor Allan Herbison for assistance establishing the fiber photometry technique and for valuable input throughout the project. We also thank Tussock Innovation and Leo van Rens (EMTech, University of Otago) for technical assistance. We thank Dr. Michel Herde, Ms. Caroline Focke, and Dr. Emmet Power for comments on an earlier version of this manuscript. This work was funded by the Prime Minister's MacDiarmid Emerging Scientist Prize and the Neurological Foundation Small Project Grant awarded to K.J.I. J.S.K. was supported by a New Zealand Lottery Health Research Postdoctoral Fellowship. K.J.I. was supported by a Sir Charles Hercus Health Research Council Fellowship.

\section{Author contributions}

J.S.K and K.J.I designed research. J.S.K performed research. S.Y.H contributed analytic tools. J.S.K and K.J.I analyzed data. All authors contributed to writing the manuscript.

\section{Competing interests}

The authors declare no competing interests.

\section{Additional information}

Supplementary information is available for this paper at https://doi.org/10.1038/s41467 019-13639-8.

Correspondence and requests for materials should be addressed to K.J.I.

Peer review information Nature Communications thanks Jamie Maguire and the other, anonymous, reviewers for their contribution to the peer review of this work. Peer reviewer reports are available.

Reprints and permission information is available at http://www.nature.com/reprints

Publisher's note Springer Nature remains neutral with regard to jurisdictional claims in published maps and institutional affiliations.

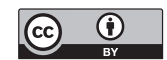

Open Access This article is licensed under a Creative Commons Attribution 4.0 International License, which permits use, sharing, adaptation, distribution and reproduction in any medium or format, as long as you give appropriate credit to the original author(s) and the source, provide a link to the Creative Commons license, and indicate if changes were made. The images or other third party material in this article are included in the article's Creative Commons license, unless indicated otherwise in a credit line to the material. If material is not included in the article's Creative Commons license and your intended use is not permitted by statutory regulation or exceeds the permitted use, you will need to obtain permission directly from the copyright holder. To view a copy of this license, visit http://creativecommons.org/ licenses/by/4.0/.

(c) The Author(s) 2019 